\title{
Stability and Sea State as Limiting Conditions for TKE Dissipation and Dissipative Heating
}

\author{
ANDREW W. SMITH AND BRIAN K. HAUS \\ Rosenstiel School of Marine and Atmospheric Science, University of Miami, Miami, Florida \\ JUN A. ZHANG \\ Cooperative Institute for Marine and Atmospheric Studies, University of Miami, \\ and NOAA/AOML/Hurricane Research Division, Miami, Florida
}

(Manuscript received 18 May 2018, in final form 12 December 2018)

\begin{abstract}
This study analyzes high-resolution ship data collected in the Gulf of Mexico during the Lagrangian Submesoscale Experiment (LASER) from January to February 2016 to produce the first reported measurements of dissipative heating in the explicitly nonhurricane atmospheric surface layer. Although typically computed from theory as a function of wind speed cubed, the dissipative heating directly estimated via the turbulent kinetic energy (TKE) dissipation rate is also presented. The dissipative heating magnitude agreed with a previous study that estimated the dissipative heating in the hurricane boundary layer using in situ aircraft data. Our observations that the 10-m neutral drag coefficient parameterized using TKE dissipation rate approaches zero slope as wind increases suggests that TKE dissipation and dissipative heating are constrained to a physical limit. Both surface-layer stability and sea state were observed to be important conditions influencing dissipative heating, with the stability determined via TKE budget terms and the sea state determined via wave steepness and age using direct shipboard measurements. Momentum and enthalpy fluxes used in the TKE budget are determined using the eddy-correlation method. It is found that the TKE dissipation rate and the dissipative heating are largest in a nonneutral atmospheric surface layer with a sea surface comprising steep wind sea and slow swell waves at a given surface wind speed, whereas the ratio of dissipative heating to enthalpy fluxes is largest in near-neutral stability where the turbulent vertical velocities are near zero.
\end{abstract}

\section{Introduction}

Within the atmospheric surface layer (ASL) that is directly adjacent to the air-sea interface, physical processes occurring at turbulent scales take place that are important to heat and momentum exchange between the ocean and directly overlying atmosphere. Dissipation of the turbulent kinetic energy within the atmospheric surface layer, which is a consequence of interfacial friction and near-surface vertical shear of the horizontal wind, results in momentum transfers to surface waves and surface currents, and a dissipative heat transfer to the atmosphere. Bister and Emanuel (1998, hereafter BE98) were the first to investigate dissipative heating, believing the term to be important to tropical cyclone

Corresponding author: Andrew W. Smith, andrew.smith@rsmas. miami.edu
(TC) maximum potential intensity. BE98 derived the term from the momentum and kinetic energy equations and inserted this term along with turbulent enthalpy flux to balance radial advection of equivalent potential temperature and vertical motion of mass associated with TC secondary circulation [BE98's Eq. (11)]. BE98 assumed all of the energy arising from the dissipation of turbulent kinetic energy is released as heat to the atmosphere, and mathematically concluded the result is a $50 \%$ increase in the enthalpy transfer coefficient and subsequent $20 \%$ increase in maximum TC wind speed.

Following the suggestions of BE98, dissipative heating parameterizations were added into more sophisticated, finescale models to produce intensity forecasts for real TCs (e.g., Zhang and Altshuler 1999; Jin et al. 2007). The dissipative heating parameterizations in these and other numerical studies (e.g., Businger and Businger 2001) have been directly related to wind speed, usually 
proportional to a surface or reference wind speed cubed. Although including the dissipative heating increased the modeled TC maximum wind speed intensity and intensity prediction accuracy of Hurricanes Andrew (Zhang and Altshuler 1999) and Irene (Jin et al. 2007), respectively, recent studies (e.g. Curcic 2015; Kieu 2015) have challenged the treatment of dissipative heating by BE9 8 because of their assumptions that (i) all dissipated TKE is transferred to the atmosphere as heat and (ii) the dissipative heating acts as an extra heat source to the atmosphere, which increases the total energy in the atmosphere-ocean system.

We present the first reported measurements of dissipative heating in the explicitly nonhurricane atmospheric surface layer and compare them with aircraft measurements from the hurricane environment during the Coupled Boundary Layers Air-Sea Transfer Experiment (CBLAST; Zhang 2010, hereafter ZH10). The results of this comparison were a primary motivation for this work, as our dissipative heating magnitudes agree with ZH10 only when the TKE dissipation rate is explicitly used (after ZH10 method). Despite the substantially different wind speed range and atmospheric state present in the two studies, we observed a similar range of dissipative heating values to those in ZH10. Therefore, we challenge the notion that the wind speed is the physical process most directly governing and constraining both TKE dissipation and dissipative heating and we agree with prior findings (e.g., Kieu 2015) that the assumptions made by BE98 are inappropriate and result in the overestimate of dissipative heating. If however, the dissipation rate and dissipative heating are not a function of wind speed but are physically constrained, what is governing their behavior? We use high-resolution ship measurements from the Lagrangian Submesoscale Experiment (LASER) in the Gulf of Mexico during January-February 2016 to investigate how the dissipation rate and dissipative heating change and how they are constrained by both the atmospheric stability and the structure of the sea surface.

\section{Theoretical background}

\section{a. Original derivation of BE98}

Dissipative heating occurs as a consequence of friction induced by the relative motion and density differences between air and water at the air-sea interface. This friction produces vertical shear of both air-side and water-side horizontal velocities and at sufficient Reynolds number, turbulent kinetic energy. In accordance with Kolmogorov (1941), the turbulence cascades to higher wavenumbers with finer scale behavior until the action of shear allows for dissipation to occur at molecular scales. BE98 built upon a previous theoretical framework for air-sea interactions in TCs (Emanuel 1986, hereafter E86) in which the ratio of enthalpy input to kinetic energy loss is critical to the maximum potential intensity TCs may achieve. To derive the dissipative heating, BE98 began with the viscous processes term in (1b) in the Navier-Stokes momentum equation:

$$
\begin{gathered}
\frac{\partial u_{i}}{\partial t}+u_{j} \frac{\partial u_{i}}{\partial x_{j}}+\frac{\partial \overline{u_{j}^{\prime} u_{i}^{\prime}}}{\partial x_{j}}=-\frac{1}{\rho} \frac{\partial p}{\partial x_{i}}+\frac{\partial}{\partial x_{j}}\left(\nu \frac{\partial u_{i}}{\partial x_{j}}\right)-g \delta_{i 3}, \\
F_{\nu}=\frac{\partial}{\partial x_{j}}\left(\nu \frac{\partial u_{i}}{\partial x_{j}}\right)
\end{gathered}
$$

where $F_{\nu}$ represents the total viscous forces, $u_{i}$ is the $i$ th component of velocity, and $\nu$ is the kinematic viscosity (theoretically) and eddy viscosity (in numerical models). Multiplying (1b) by $u_{i}$ for each $j$ direction and manipulating the resulting expression yields

$$
F_{\nu}=\frac{\partial}{\partial x_{j}}\left[\nu \frac{\partial}{\partial x_{j}}\left(\frac{1}{2} u_{i}^{2}\right)\right]-\nu\left(\frac{\partial u_{i}}{\partial x_{j}}\right)^{2} .
$$

The terms in (2) represent the diffusion and dissipation of kinetic energy, respectively. Dissipation of kinetic energy is quadratically related to shear of the mean flow. The second term in (2) could be recast in two dimensions when considering eddy viscosities acting in zonal and meridional directions, but such an expression is valid in the flow interior not at the surface. However, the vertical shear of the horizontal wind that is important in the dissipation process can be related to a surface shear stress

$$
\left.\frac{\tau}{\rho} \sim \nu \frac{\partial u_{i}}{\partial x_{3}}\right|_{z=0}=C_{D} u_{i} \sqrt{u_{1}^{2}+u_{2}^{2}},
$$

where $C_{D}$ is the drag coefficient, $\rho$ is the density, $\tau$ is the surface stress, and the $u_{1}$ and $u_{2}$ terms are the first and second components, $u$ and $v$, respectively. Here, the surface shear stress is equivalent to the bulk aerodynamic product of the drag coefficient $C_{D}$, the zonal velocity, and the wind speed. BE98 parameterize the frictional dissipation in their axisymmetric TC numerical model scaled by the height $h$ above sea level of their lowest model grid point as

$$
\frac{C_{D}}{h}\left(u_{1}^{2}+u_{2}^{2}\right)^{3 / 2}
$$

and using (3b) a dissipative heating term was summed with the turbulent enthalpy flux to balance radial advections of equivalent potential temperature in the secondary circulation of the BE98 model: 


$$
c_{p} \psi_{b} \frac{\partial \ln \theta_{e}}{\partial r}=\frac{1}{T_{S}}\left(\tau_{k_{0}}+\frac{1}{g} \int_{p_{b}}^{p_{0}} D d p\right),
$$

where the dissipative heating is the well-mixed boundary layer integrated dissipation rate,

$$
\frac{1}{g} \int_{p_{b}}^{p_{0}} D d p=\mathscr{D}_{U}=\rho C_{D}|\mathbf{V}|^{3}
$$

and is equal to the product of air density, drag coefficient, and the cube of the wind speed.

\section{b. Revised theory from recent studies}

While the results from a comparison of dissipative heating measurements in explicitly different atmospheric surface layer environments is a motivating impetus for our work, the focus of our investigation is the dissipation rate of turbulent kinetic energy. Nevertheless, we must give a complete account of the dissipative heating, which includes the reassessment of the conclusions put forward by BE98 and our knowledge to date. For the full details, we direct the reader to Kieu (2015).

Briefly, Kieu (2015) argue that the BE98 representation of dissipative heating in tropical cyclones is questionable because it (i) treats dissipative heating as an external heat input that adds energy to the atmosphere and (ii) assumes a perfect efficiency of energy transfer purely to the atmosphere as dissipative heat. We explain both of these issues in the following matter, beginning with the problem of external heat input.

The energy equation for a Lagrangian fluid parcel (cf. Holton 2004) is

$$
\Gamma=C_{v} T+\frac{1}{2}|\mathbf{U}|^{2}+g z,
$$

where the three terms on the right-hand side of (5a) are the enthalpy, kinetic energy, and potential energy (or geopotential), respectively. Constructing the material derivative that describes the time rate-of change of such a parcel gives us a prognostic equation for the energy that must be conserved:

$$
\begin{aligned}
\rho \frac{D \Gamma}{D t}= & \underbrace{-\nabla \cdot(p U)}_{1}+\underbrace{\rho J}_{2}-\underbrace{\sum_{i} \frac{\partial \kappa_{i}}{\partial x_{i}}}_{3} \\
& +\underbrace{\sum_{i} \sum_{j} \frac{\partial\left(\sigma_{i j} u_{i}\right)}{\partial x_{j}}}_{4}-\underbrace{\sum_{i} \sum_{j} \frac{\partial\left(\tau_{i j} u_{i}\right)}{\partial x_{j}}}_{5} .
\end{aligned}
$$

On the right-hand side of $(5 b)$, term 1 is the energy generation due to pressure-gradient force, term 2 is the external heat sink/source term, term 3 is the eddy heat flux $\kappa_{i}=\overline{\rho u_{i}^{\prime} T^{\prime}}$, term 4 is the molecular viscous dissipation, and term 5 represents the energy changes due to eddy momentum flux of wind component $i$ acting in direction $j, \tau_{i j}=\overline{\rho u_{i}^{\prime} u_{i}^{\prime}}$. Since both enthalpy and momentum are included in $(5 \mathrm{a})$, the thermodynamic equation can be isolated by subtracting off the momentum equation:

$$
\rho \frac{D}{D t}\left(\frac{1}{2}|\mathbf{U}|^{2}+g z\right)=-\mathbf{U} \cdot \nabla p+\sum_{i} \sum_{j} u_{i} \frac{\partial \sigma_{i j}}{\partial x_{j}}-\sum_{i} \sum_{j} u_{i} \frac{\partial \tau_{i j}}{\partial x_{j}}
$$

leaving the thermodynamic equation:

$$
\begin{aligned}
\rho \frac{D\left(C_{v} T\right)}{D t}= & -p \nabla \cdot \mathbf{U}+\rho J-\sum_{i} \frac{\partial \kappa_{i}}{\partial x_{i}} \\
& +\sum_{i} \sum_{j} \sigma_{i j} \frac{\partial u_{i}}{\partial x_{j}}-\sum_{i} \sum_{j} \tau_{i j} \frac{\partial u_{i}}{\partial x_{j}} .
\end{aligned}
$$

If the eddy momentum flux is prescribed the form $\tau_{i j}=-\nu \partial u_{i} / \partial x_{j}$, the last term in (5d) is

$-\sum_{i} \sum_{j} \tau_{i j} \frac{\partial u_{i}}{\partial x_{j}}=-\sum_{i} \sum_{j}\left(-\nu \frac{\partial u_{i}}{\partial x_{j}}\right) \frac{\partial u_{i}}{\partial x_{j}}=\sum_{i} \sum_{j} \nu\left(\frac{\partial u_{i}}{\partial x_{j}}\right)^{2}$,

which is functionally identical to BE98's equation for dissipative heating in the interior of the fluid flow [second term in (2); also BE98's (4)]. So in actuality, the dissipative heating is not an extra or external heat source, but instead acts internally within the fluid flow to prevent violation of energy conservation.

The second major issue in BE98's theoretical framework for dissipative heating is what Kieu (2015) denotes as the "no-work to environment" problem. By assuming all of the frictional work done when turbulent kinetic energy is dissipated is converted into dissipative heating within the atmospheric surface layer, the efficiency of the system is treated as perfect. In invoking such an assumption, TCs for example could reuse all of the frictional work with no loss to the surrounding environment. This frictional work imposed by the well-mixed boundary layer on the atmospheric surface layer at the atmospheric surface layer height $z=h$ [see Kieu (2015)'s Fig. 2] is considered within a cylindrical fluid volume and is given by

$$
F_{\text {diss }}=-\int_{\Omega}\left[\sum_{i} \sum_{j} \frac{\partial\left(\tau_{i j} u_{i}\right)}{\partial x_{j}}\right] d V \approx-\int_{\Omega}\left[\sum_{i=1,2} \frac{\partial\left(\tau_{i j} u_{i}\right)}{\partial z}\right] d V .
$$

Vertical shear of horizontal winds dominate the eddy momentum flux $\tau_{i j}$, which becomes $\tau_{i 3}$ and (7a) simplifies to 


$$
F_{\mathrm{diss}} \approx-2 \pi C_{D} \int_{0}^{R_{0}} \rho|V|^{3} r d r
$$

which represents the full frictional work and is equivalent to (4b). This is not, however, the amount that is transferred to the atmosphere as dissipative heating because the atmospheric surface layer uses this work to (i) internally warm the layer (dissipative heating), (ii) contribute to surface ocean wave growth and subsequently sea spray, and (iii) do work on the upper ocean layer such that energy may be conveyed to the surface currents. An estimate of the fractioning of $F_{\text {diss }}$ into these sinks is described in Richman and Garrett (1977) and we will not discuss this here.

Both of the assumptions we mentioned here result in either inappropriate addition of or overestimation of energy being transferred to the atmosphere. ZH10 used high-resolution measurements of velocity within the hurricane boundary layer in CBLAST to determine the dissipative heating using the TKE dissipation rate, and demonstrate that (4b) from BE98 overestimates the dissipative heating. We will show later that our dissipative heating measurements, which come from an explicitly nonhurricane atmospheric surface layer, clearly corroborate the discrepancy outlined by ZH10.

\section{c. Dissipative heating from TKE dissipation rate}

From ZH10's Eq. (1), the turbulent kinetic energy budget equation is

$$
\begin{aligned}
\frac{D e}{D t}= & \underbrace{-\overline{u^{\prime} w^{\prime}} \frac{\partial \bar{u}}{\partial z}}_{1}-\underbrace{\overline{v^{\prime} w^{\prime}} \frac{\partial \bar{v}}{\partial z}}_{2}+\underbrace{\left(\frac{g}{\bar{\theta}}\right) \overline{w^{\prime} \theta^{\prime}}+0.61 g \overline{w^{\prime} q^{\prime}}}_{3} \\
& -\underbrace{\frac{\partial \overline{w^{\prime} e}}{\partial z}}_{4}-\underbrace{\frac{1}{\partial \frac{\partial w^{\prime} p^{\prime}}{\partial z}}}_{5}-\underbrace{\varepsilon}_{6},
\end{aligned}
$$

where $e=\left(\overline{u^{\prime 2}}+\overline{v^{12}}+\overline{w^{12}}\right) / 2$, and on the right-hand side, terms 1 and 2 are the zonal and meridional shear production, respectively, term 3 is the dry and moist buoyancy production, term 4 is the turbulent transport, term 5 is the nonhydrostatic turbulent pressure work, and term 6 is the dissipation rate of TKE.

The dissipation rate of TKE is integrated over the surface-layer height $z_{1}$ and multiplied by the air density $\rho$ to yield the dissipation-rate-dependent dissipative heating presented in $\mathrm{ZH} 10$ :

$$
D_{\varepsilon}=\rho \bar{\varepsilon} z_{1} .
$$

ZH10 prescribe $z_{1}=200 \mathrm{~m}$ to be the surface-layer height, with the assumption that all of the TKE dissipation and dissipative heating occur below that height.
While the dissipation-rate measurements from ZH10 have been found to be independent of the aircraft height $\left(R^{2}=-0.049\right)$, the choice of $z_{1}$ will linearly change the dissipative heating $D_{\varepsilon}$ determined using (9). Although Businger and Businger (2001) have found dissipative heating to be insensitive to the choice of $z_{1}$ for wind speeds as high as $50 \mathrm{~ms}^{-1}$, the true surface-layer height over the Gulf of Mexico during LASER is very likely shallower than that in the hurricane environments sampled by $\mathrm{ZH} 10$. Therefore, we choose $z_{1}=125 \mathrm{~m}$ based on multidecadal ECMWF reanalysis climatological-average planetary boundary layer (PBL) heights for December-February presented in Von Engeln and Teixeira (2013, see their Fig. 5). The specific value was taken from the region south of the central Gulf of Mexico near the LASER study site, and we assume that the surface-layer height is $10 \%$ of the PBL height. We acknowledge that careful selection of $z_{1}$ is a caveat of (9), and believe that basing the choice for $z_{1}$ off of the aforementioned climatology reduces ambiguity and improves the robustness of the dissipative heating measurements.

\section{Data}

\section{a. Field experiment background}

LASER was conducted January-February 2016 by investigators from the Consortium for Advanced Research on Transport of Hydrocarbons in the Environment (CARTHE) group, as part of the Gulf of Mexico Research Initiative (GoMRI) to investigate marine, biological, and physical effects of the Deepwater Horizon $(D W H)$ oil spill (2010). LASER was motivated by data and preliminary findings on near-surface ocean transport from large-scale currents and eddies to submesoscale fronts, collected during the Grand Lagrangian Deployment (GLAD) experiment in August 2012 (e.g., Olascoaga et al. 2013; Poje et al. 2014; Beron-Vera and LaCasce 2016). In particular, LASER was a coordinated effort to investigate crude oil transport near to the DeSoto Canyon region of the continental shelf. Figure 1a shows the study region overlain with the vessel track of the R/V F.G. Walton Smith (FGWS), operated by the University of Miami Rosenstiel School of Marine and Atmospheric Sciences (RSMAS). Data for this study were taken from instruments mounted onto and on board the $F G W S$, and are described in further detail in the next section.

\section{b. Instrumentation and measurements}

Surface-layer atmospheric and both in situ and remote surface ocean measurements were collected on the FGWS using a combination of fixed-mount digital 

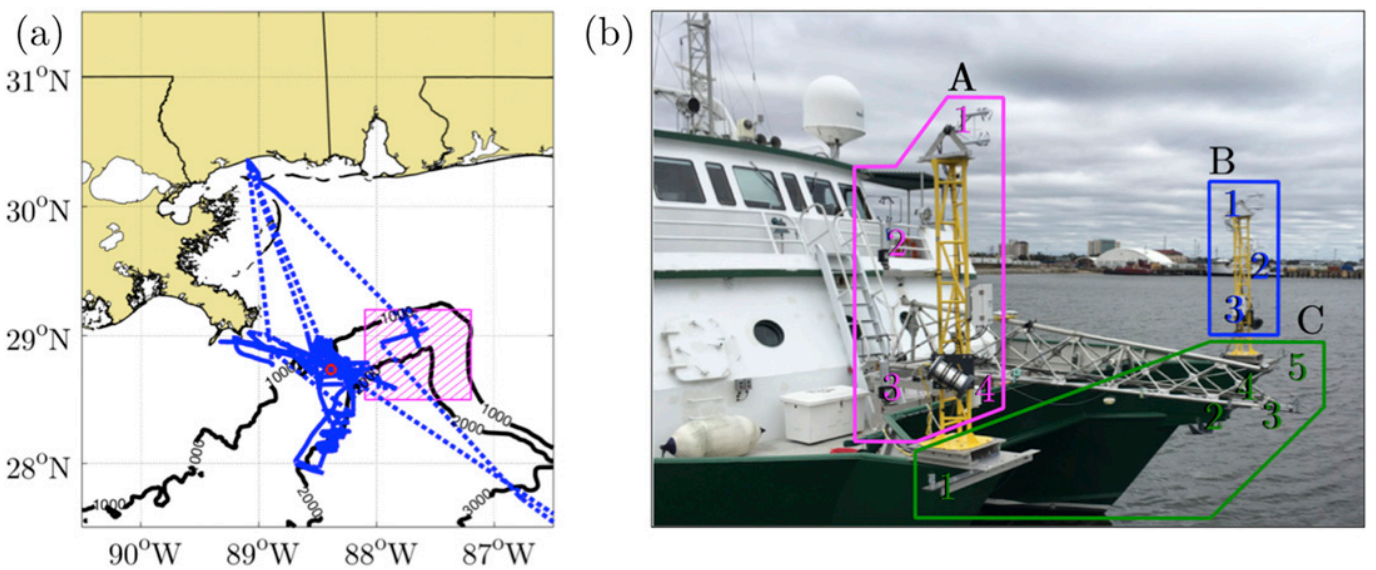

FIG. 1. (a) Primary site for the LASER experiment in the Gulf of Mexico. Solid blue lines show the recording periods and track of the R/V F.G. Walton Smith. The red circle is the location of the Deepwater Horizon oil rig. The magenta hatched square covers the DeSoto Canyon region. Black contours with numbers are the isobaths $(\mathrm{m})$. (b) Bow instrumentation on the F.G. Walton Smith during LASER. Group A (B) refer to the starboard (port) flux towers, equipped with IRGASON, RMY, and Rotronic RH/AT sensor numbered in that order on each tower. Group $C$ refers to the array of five Senix ultrasonic distance meters.

sensors, direct water sampling instruments, and a marine Doppler radar (MDR). With the exception of port calls and localized maneuvering or small-boat operations as needed, the ship was under way in open ocean with nonzero forward speed $0<U_{\text {vessel }} \leq 4.67 \mathrm{~m} \mathrm{~s}^{-1}$ when data were collected. To guarantee open-ocean conditions, we used the ETOPO1 1-min arc global relief model topography dataset from the NOAA/National Centers for Environmental Information to exclude data taken over waters less than or equal to $100 \mathrm{~m}$, or $6.6 \%$ of the average depth of the Gulf of Mexico $(1524 \mathrm{~m})$. Figure $1 \mathrm{~b}$ shows the configuration of highfrequency $(16 \mathrm{~Hz})$ instruments on the $F G W S$ bow, consisting of two flux towers on each prow, and a center truss. The flux towers consisted of two triangular, metal scaffolds affixed with 1) one Campbell Scientific IRGASON integrated sonic anemometergas analyzer capable of measuring 3D velocity, sonic temperature, IR-gas analyzer derived water vapor and carbon dioxide concentrations, and air pressure; and 2) two RM Young sonic anemometers (one lower, one upper) measuring $3 \mathrm{D}$ velocity and sonic temperature. Additionally, the starboard prow flux tower included 3) a Rotronic relative humidity ( $\mathrm{RH}$ )-air temperature sensor equipped with a radiation and moisture shield. The center truss, attached to the bow deck, included an array of three Senix ultrasonic distance meters (UDMs) plus one on each prow (total of five) sampling at $16 \mathrm{~Hz}$. Exact heights of bow instrumentation from mean water level are given in Table 1. A Seabird Scientific SBE 45 microthermosalinograph (MicroTSG) routing surface seawater samples from intakes at the bow of the FGWS to the fore-stern wet laboratory collected sea surface temperature (SST), salinity (SSS), and pressure at $6-\mathrm{Hz}$ frequency. A single X-band MDR was mounted above the wheelhouse, which collected approximately 30-min circular swaths of backscatter data with $7.5 \mathrm{~m} \times 7.5 \mathrm{~m}$ resolution extending a radius of about $3 \mathrm{~km}$ each direction. Near-surface current data were also available for each MDR swath, the construction and details of which are described in Lund et al. (2015). Ship motion (i.e., roll, pitch, yaw, surge, sway, and heave) was measured using a motion measurement package including 1) one Columbia Research Laboratory SA-307HPTX

TABLE 1. R/V F.G. Walton Smith bow instrumentation heights. For the center column "P" denotes port prow, "S" denotes starboard prow, and "CT" denotes center truss. For the UDMs, "-O" denotes "outboard."

\begin{tabular}{llc}
\hline \hline \multicolumn{1}{c}{ Instrument } & $\begin{array}{c}\text { Fixed-mount } \\
\text { location }\end{array}$ & $\begin{array}{c}\text { Height } \\
(\mathrm{m})\end{array}$ \\
\hline $\begin{array}{l}\text { Motion measurement package } \\
\text { (QRS11 and SA-307HPTX) }\end{array}$ & & 3.986 \\
IRGASON & $\mathrm{P}$ & 5.950 \\
IRGASON & $\mathrm{S}$ & 5.955 \\
RMY (lower) & $\mathrm{P}$ & 4.155 \\
RMY (upper) & $\mathrm{P}$ & 5.190 \\
RMY (lower) & $\mathrm{S}$ & 4.165 \\
RMY (upper) & $\mathrm{S}$ & 5.185 \\
ROTRONIC RH/AT & $\mathrm{S}$ & 3.685 \\
SENIX UDM & $\mathrm{P}$ & 3.150 \\
SENIX UDM & $\mathrm{CT}-\mathrm{P}$ & 3.325 \\
SENIX UDM & $\mathrm{CT}-\mathrm{O}$ & 3.325 \\
SENIX UDM & $\mathrm{CT}-\mathrm{S}$ & 3.325 \\
SENIX UDM & $\mathrm{S}$ & 3.150 \\
\hline
\end{tabular}


accelerometer and 2) one Systron Donner QRS11 angular rate gyro.

\section{c. Data limitations and motion correction}

Despite the high frequency of sampling and diversity of measurements, data collection was subject to the assumptions of functioning instrumentation, operating while under way in open ocean, and involved measurements which were taken from the moving reference frame of the vessel. To eliminate the platform motion from measurements and their subsequent derived quantities, motion correction was applied in a preprocessing stage to the data. Motion correction has been performed on data from aircraft (e.g., Miyake et al. 1970), ships (Fujitani 1985), and buoys (Dugan et al. 1991) using knowledge of the axial and angular motion of the platform or the instrument. Fortunately, since pitch $\theta$, roll $\phi$, and yaw $\psi$ were measured by the $F G W S$ motion package the application of a simple motion-correction algorithm outlined in Anctil et al. (1994) permits us to retrieve true fixed-mount instrument data independent to data quality flagging performed by the instrument data acquisition systems. The relative-absolute motion transformation matrix $\mathbf{T}_{B E}$ (Anctil et al. 1994) is

$$
\mathbf{T}_{B E}=\left(\begin{array}{ccc}
\cos \theta \cos \psi & \sin \phi \sin \theta \cos \psi-\cos \phi \sin \psi & \cos \phi \sin \theta \cos \psi+\sin \phi \sin \psi \\
\cos \theta \sin \psi & \sin \phi \sin \theta \sin \psi+\cos \phi \cos \psi & \cos \phi \sin \theta \sin \psi-\sin \phi \cos \psi \\
-\sin \theta & \sin \phi \cos \theta & \cos \phi \cos \theta
\end{array}\right)
$$

where the subscript $B E$ refers to the transformation between the platform $B$ accelerations $\mathbf{u}_{B}$ and Earth reference frame accelerations $\mathbf{u}_{E}$. Gravitational acceleration of the platform is given by

$$
\mathbf{g}_{B}=\left(\begin{array}{lll}
0 & 0 & -g
\end{array}\right)^{\mathrm{T}},
$$

with $g=9.81 \mathrm{~m} \mathrm{~s}^{-2}$ and the superscript $\mathrm{T}$ notation denoting the matrix transpose. Including (10), $\mathbf{u}_{E}$ can be found via

$$
\mathbf{u}_{E}=\mathbf{T}_{B E} \mathbf{u}_{B}+\mathbf{T}_{B E} \int\left(\mathbf{a}_{B}+\mathbf{g}_{B}\right) d t+\mathbf{\Omega} \times \mathbf{T}_{B E} \mathbf{L}_{B}
$$

where the angular rotation vector $\boldsymbol{\Omega}$ is

$$
\mathbf{\Omega}=\left(\begin{array}{c}
-\dot{\theta} \sin \psi+\dot{\phi} \cos \theta \cos \psi \\
\dot{\theta} \cos \psi+\dot{\phi} \cos \theta \sin \psi \\
\dot{\psi}-\dot{\phi} \sin \theta
\end{array}\right)
$$

with dots indicating the time derivative of the dotted quantity. The cross-product $\mathbf{\Omega} \times \mathbf{T}_{B E} \mathbf{L}_{B}$ denotes the angular acceleration given the physical distance between the motion package and the instrument itself denoted by $3 \mathrm{D}$ vector $\mathbf{L}_{B}$.

Quality-control flags were implemented on raw data collected on the FGWS bow, including the turbulent velocity, temperature, pressure, and humidity measurements from the flux towers as well as the truss and prow UDM data. The resulting subsample of the original dataset only included 1) measurements where the difference of the mean wind direction and ship heading (HMWD) was within an arc of $20^{\circ}$ to port or starboard of $0^{\circ}$ (ship moving directly into the wind), and 2 ) reduced the five UDM array to a 3 UDM triangle based on elevation spectra error characteristics described in section 4c. Any flux data for which HMWD was greater than $\pm 20^{\circ}$ was treated as possibly subject to flow distortion and not included. As a secondary precaution, only turbulent velocity and sonic temperature data from the highest two IRGASON sonic anemometers (A1 and B1; see Fig. 1b) and the middle two RM Young sonic anemometers (A2 and B2) were used. Inclusion of turbulent measurements at minimum two different heights was required to compute vertical gradients in the turbulent kinetic energy budget [see (8)]. An overview of meteorological and oceanographic conditions during LASER is presented in Fig. 2.

\section{Analysis methodology}

\section{a. Dissipation rate and dissipative heating}

The dissipation rate of TKE was computed using the motion-corrected $3 \mathrm{D}$ wind speed $U_{a}=\sqrt{u_{a}^{2}+v_{a}^{2}+w_{a}^{2}}$, and the relationship put forth by Kolmogorov (1941) describing the energy cascade of TKE from low toward high wavenumber with the following form:

$$
S(k)=\alpha \varepsilon^{2 / 3} k^{-5 / 3},
$$

where $S(k)$ is the TKE power spectrum, or power spectral density of the energy. Rearranging (14) and transforming from wavenumber to frequency space, we get the dissipation rate as determined in $\mathrm{ZH} 10$ :

$$
\varepsilon=\alpha^{-3 / 2} \frac{2 \pi f}{U_{a}}\left[f S_{u u}(f)\right]^{3 / 2},
$$

with the Kolmogorov constant, $\alpha=0.5$ (Sreenivasan 1995), the frequency denoted by $f$, and $S_{u u}(f)$ the velocity spectrum. Velocity spectra were constructed from 
(a)

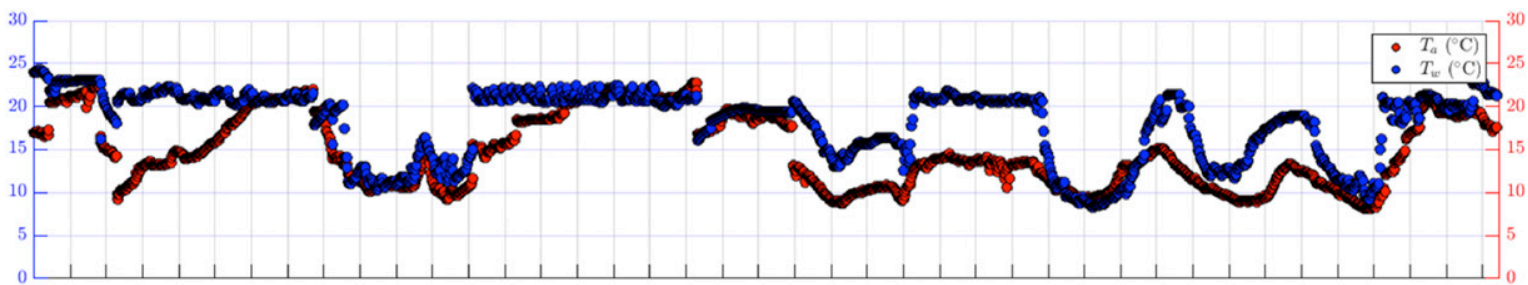

(b)

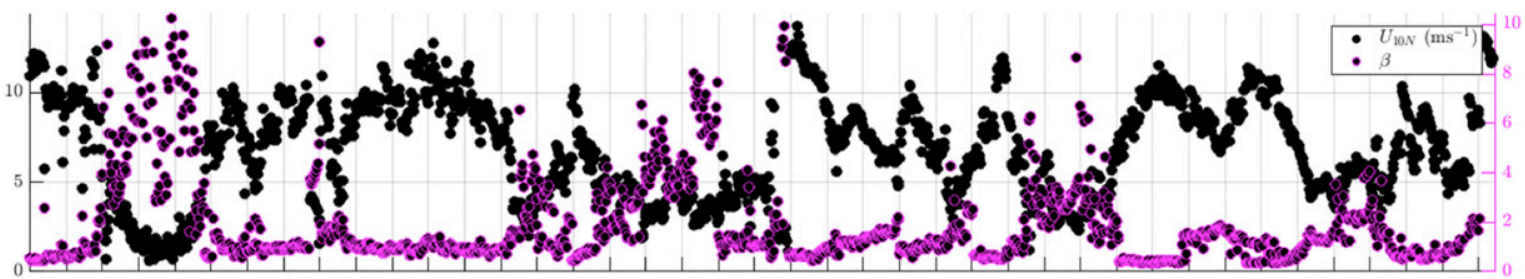

(c)

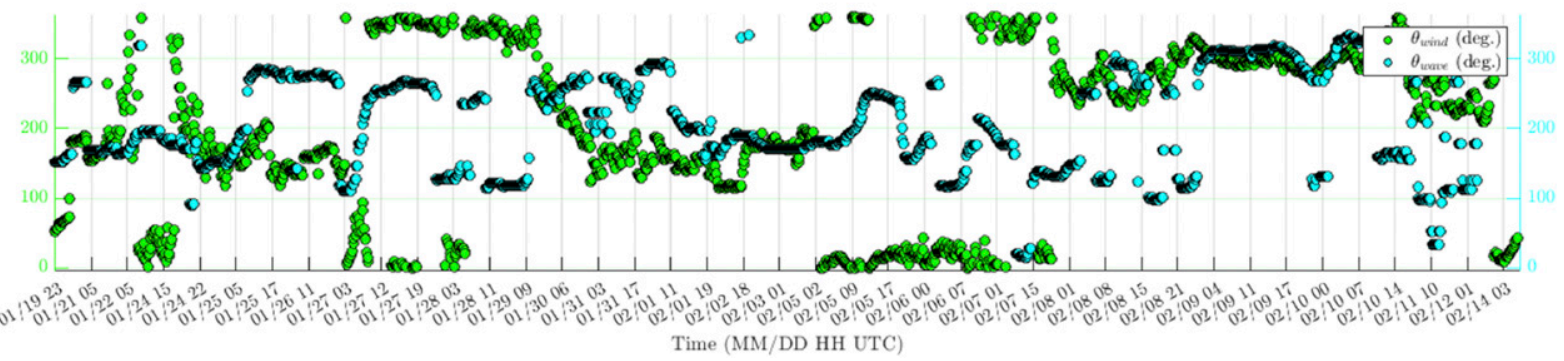

FIG. 2. Summary of meteorological and oceanographic conditions during LASER, including (a) air and water temperature, (b) 10-m neutral wind speed and wave age, and (c) mean wind and wave direction.

each 10-min along-flow (bow-stern axis) velocity time series through a Fourier transform on the time series. A Blackman-Harris windowing scheme was also used to average elemental frequencies along the spectrum, producing the final size of the spectrum. We chose the number of frequencies to average $N_{\mathrm{FA}}=16$ to sufficiently represent the spectra at higher frequencies while limiting noise. The velocity spectra $S_{u u}(f)$ are confined to the $2-4-\mathrm{Hz}$ range of frequencies based on agreement with the inertial-subrange slope $f^{-5 / 3}$. Velocity spectra were rejected if the error of their slope in this frequency band exceeded a specified error threshold for both anemometers A1 and B1. Ultimately, we selected an error threshold of $55 \%$ based on the derivative of curve describing the amount of data retained versus the error threshold value. Based on this criterion, 858 of 1617 or $53.1 \%$ of the 26-day dataset was retained. Figure 3 shows all the retained velocity spectra collected by FGWS during LASER, as well as the median velocity spectra for (Fig. 3a) B1 and (Fig. 3b) A1 anemometers. The sensitivity of data retained given a certain error threshold is described by the curve plotted in (Fig. 3c). The two median spectra are composed of the median power spectral density at each frequency interval $d f$ from the minimum frequency $f_{\min }=0.01 \mathrm{~Hz}$ to the Nyquist frequency $f_{N}=8 \mathrm{~Hz}$. The median spectra compare well to each other, with power spectral density values in the range of $10^{-7}-10^{2}$, and the slope of the $2-4-\mathrm{Hz}$ band in the median data appear similar to the expected slope of $f^{-5 / 3}$. The dissipative heating was computed using two formulations shown in (4b) and (9), the formulations of BE98 and ZH10, respectively. In both methods, the moist air density, $\rho$, was measured directly by anemometer A4 (see Fig. 1b) on the starboard prow flux tower. Both the 10 -m neutral values of $C_{D}$ and $U\left(C_{D 10 \mathrm{~N}}\right.$ and $U_{10 \mathrm{~N}}$, respectively) were calculated using a bulk method based on the wind speed and the height of the measurements from anemometer A1 (see Fig. 1b) and stability following Smith (1988).

\section{b. Eddy-covariance fluxes and atmospheric stability}

Having sampled at high-frequency the various atmospheric variables, we capture turbulent motions and therefore make use of the eddy-covariance method (e.g., Burba and Anderson 2007) to compute the fluxes of sensible heat, latent heat, and momentum. This technique is 

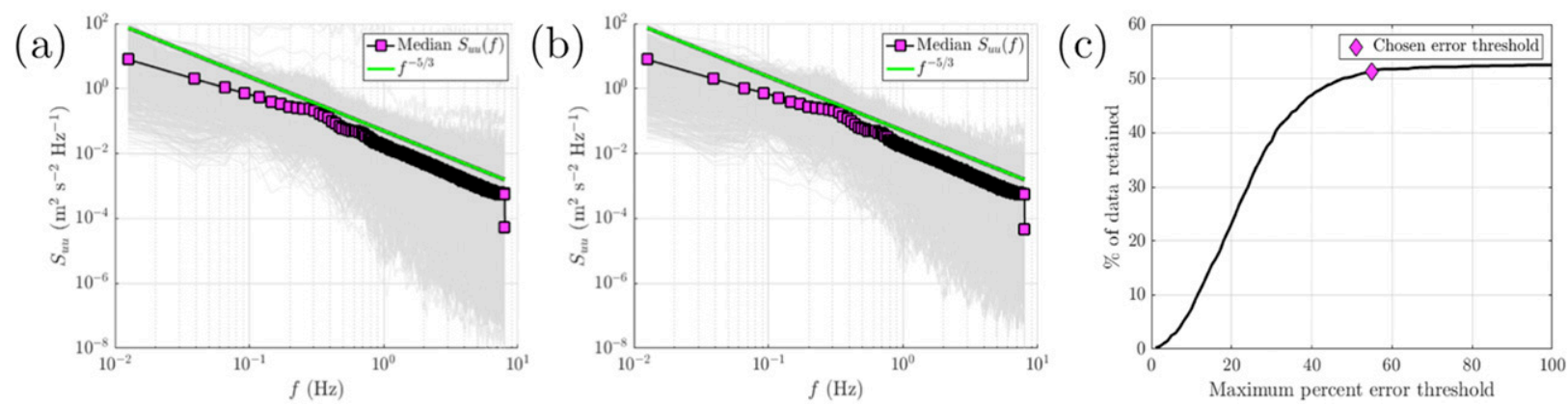

FIG. 3. Zonal velocity spectra $\left(\mathrm{m}^{2} \mathrm{~s}^{-2} \mathrm{~Hz}^{-1}\right)$ derived from 10-min velocity measurements from the (a) port and (b) starboard IRGASON anemometers during LASER. The gray solid lines shows the individual spectra. The per-frequency-bin median is shown by the magenta squares. The solid lime green line is the $f^{-5 / 3}$ slope line for the inertial subrange. (c) The percentage of total data falloff for a given inertial-subrange error threshold. The magenta diamond indicates the chosen error threshold we use.

also used to determine the eddy transport and pressure work terms in the TKE budget [terms 4 and 5 in (8)]. The quotient of shear and buoyancy production terms from the TKE budget, which also involve eddy covariance fluxes, is used to determine atmospheric stability. The eddy-covariance method involves a Reynolds decomposition of vertical velocity and a scalar quantity of interest, which are then both detrended. The covariance represented by the product of the perturbation of the two terms is then averaged over a suitable time window. We use a time average of $10 \mathrm{~min}$, however averages of 15,30 , and $60 \mathrm{~min}$ are also commonly used. Time averages of greater than $60 \mathrm{~min}$ are not advisable for turbulent measurements because of the probable loss of stationarity (Large and Pond 1981).

The sensible and latent heat fluxes take the following form:

$$
\begin{aligned}
& H_{S}=\rho c_{p d} \overline{w^{\prime} T^{\prime}}, \\
& H_{L}=\rho L_{v} \overline{w^{\prime} q^{\prime}},
\end{aligned}
$$

where $c_{\mathrm{pd}}=1004.67 \mathrm{~J} \mathrm{~kg}^{-1} \mathrm{~K}^{-1}$ is the specific heat capacity of dry air at constant pressure, $q$ is the specific humidity at saturation, $T$ is the air temperature, and $L_{v}=2.5 \times 10^{6} \mathrm{~J} \mathrm{~K}^{-1}$ is the latent heat of vaporization. Following (1) from Zhang et al. (2011), we define the momentum flux $\tau$ as

$$
\tau=\rho\left(-\overline{w^{\prime} u^{\prime}}-\overline{w^{\prime} v^{\prime}}\right) .
$$

With the dissipation rate determined, we need the TKE production and transport terms to complete the righthand side of the TKE budget given in (8). Shear production was computed using the zonal eddy-covariance momentum flux and mean wind speed measurements from anemometers A1 and A2 (see Fig. 1b). The vertical gradient of measurements between the two anemometers gave the mean zonal wind shear. Because of the quality control applied to turbulent flux data to account for good HMWD, only term 1, rather than both terms 1 and 2 , in (8) was retained. Therefore, we can simplify the shear production of TKE to

$$
S=-\overline{u^{\prime} w^{\prime}} \frac{\partial \bar{u}}{\partial z}
$$

Buoyant processes can either produce or consume TKE depending on the stability of the atmosphere, and contributions to the buoyant production term in the TKE budget can come from both dry and moist air. Sonic velocity and temperature measurements from anemometer A1 were used to calculate the eddy heat flux terms including specific humidity $q$. The perturbation and mean potential temperatures were calculated using the familiar expression,

$$
\theta=T\left(\frac{p_{0}}{p}\right)^{R_{d} / c_{p}},
$$

where $p_{0}=1000 \mathrm{hPa}$ is the reference pressure, $R_{d}=$ $287 \mathrm{~J} \mathrm{~kg}^{-1} \mathrm{~K}^{-1}$ is the dry air gas constant, and $c_{p}=$ $c_{p d}\left(1+0.84 r_{v}\right)$ is the specific heat capacity at constant pressure. The water vapor mixing ratio $r_{v}$, a function of the vapor pressure $e$ and total pressure $p$, is

$$
r_{v}=\frac{0.662 e}{p-e} .
$$

We now individually define the buoyancy production of TKE as the sum of the dry and moist terms:

$$
B=\frac{g}{\bar{\theta}} \overline{w^{\prime} \theta^{\prime}}+0.61 g \overline{w^{\prime} q^{\prime}} .
$$

Vertical redistribution of TKE is accomplished by perturbations in vertical velocity and pressure that are 
associated with convection, gravity waves, and buoyancy fluctuations (e.g., Edson and Fairall 1998). Since the pressure was only measured via the IRGASON pressure cell (anemometers A1 and B1; see Fig. 1b), a vertical derivative of pressure could not be constructed and the pressure work [term 5 in (8)] was determined from the residual of the other TKE budget terms. So the two transport terms are

$$
\begin{aligned}
& T=-\frac{\partial \overline{w^{\prime} e^{\prime}}}{\partial z}, \\
& P=S-B-T-\varepsilon .
\end{aligned}
$$

Finally, the Monin-Obukhov atmospheric stability parameter $\zeta$ is related to the negative ratio of buoyancy and shear:

$$
\begin{aligned}
\zeta=z \times\left(-\frac{S}{B}\right)^{-1} & =z \times\left(-\frac{g \overline{\bar{\theta}} \overline{w^{\prime} \theta^{\prime}}+0.61 g \overline{w^{\prime} q^{\prime}}}{-\overline{u^{\prime} w^{\prime}} \frac{\partial \bar{u}}{\partial z}}\right) \\
& =-\frac{g \kappa z \overline{w^{\prime} \theta_{v}^{\prime}}}{u_{*}^{3} \overline{\theta_{v}}}=\frac{z}{L},
\end{aligned}
$$

where $\kappa$ is the von Kármán constant. The virtual potential temperature, which includes both dry and moist thermodynamics, replaces the sum of sensible and latent contributions to buoyancy in (19a) and is defined by

$$
\theta_{v}=\theta(1+0.61 q)
$$

As a result of the definition in (19a), shear production of TKE homogenizes the surface layer and works against existing density stratification in the atmosphere while buoyancy production either homogenizes or stratifies the atmospheric surface layer based on the sign of the perturbations of equivalent potential temperature defined by (19b).

\section{c. Sea-state determination}

Subjection of the sea surface to physical forcing by the wind, particularly in the open ocean where fetch may be large and uninterrupted, allows for potentially large energy transfer between the atmosphere and the ocean. Roughening of the waves on the sea surface, regardless of whether the waves are locally growing by wind or are swell may influence the flow of air in the surface layer as well as the rate of TKE dissipation. Furthermore, since previous studies have shown that the TKE budget terms can balance and contribute differently in swell as compared with wind sea (e.g., Sjöblom and Smedman 2002; Högström et al. 2009), we have segregated the sea states using water surface elevation spectra from the $F G W S$ bow UDM array (C1-5; see Fig. 1b) using a three-step process. Each 10-min data segment of water surface elevation spectra was quality controlled, then the wavelet directional method (WDM; Donelan et al. 1996) was implemented, and finally the Pierson and Moskowitz (1964) criterion, $c_{p} / U_{10 \mathrm{~N}}>1.2$ for swell, was applied. Discrimination of the sea states using wind speed and a coefficient of 1.2 is demonstrated in Potter (2015, see their Fig. 1).

Quality control of the five UDMs began by eliminating spectrum data for frequencies less than $0.0667 \mathrm{~Hz}$ (a period of 15s) as being an unlikely region to find the spectral peak considering the fetch of the Gulf of Mexico (W. M. Drennan 2017, personal communication). In order for the WDM to be implemented properly and to provide the most useful output, a minimum-of-three group of geometrically arranged signals was needed. The instruments also needed to be separated sufficiently in both along-bow and across-bow axes to provide accurate estimates of wave direction across a range of wave scales. Given their large $(10.22 \mathrm{~m})$ separation distance, the median of the spectra from the port and starboard prow UDM (C1 and C5), computed for each frequency bin, was used as a truth measurement of wave data not contaminated by signal cross-talk resulting from UDMs being too close together. We followed this by determining which UDMs in the center-truss triplet (C2-C4; see Fig. 1b) had "runaway error," frequency by frequency for each UDM against the median spectra we took as truth. The runaway error term refers to increasing error rapidly at some (critical) frequency, such that the spectrum no longer follows the slope of the truth spectrum at frequencies beyond this critical frequency. UDMs with this behavior were flagged as suspect, and the two prow spectra and one of the center triplet of $\mathrm{C} 2-\mathrm{C} 4$ spectra were flagged as good for each 10-min segment of wave data.

While the exact details of WDM as described in (Donelan et al. 1996) are left to the reader, we present the basic steps. We first despiked the three good post-quality-controlled water surface elevation spectra, and defined the relative positions and spacing in the along-bow and across-bow axis for the triangle created by the three UDMs. From the array of water surface elevation spectra, a wave energy amplitude matrix was constructed using the Morlet wavelet option, and then transformed into a $2 \mathrm{D}$ directional wavenumber-frequency spectrum constrained by a directional resolution of $5^{\circ}$ and wavenumber resolution of 0.1 over the range of wavenumbers from 0.1 to 2. The location of the maximum spectral density in the 2D spectrum provided the dominant wave direction, 

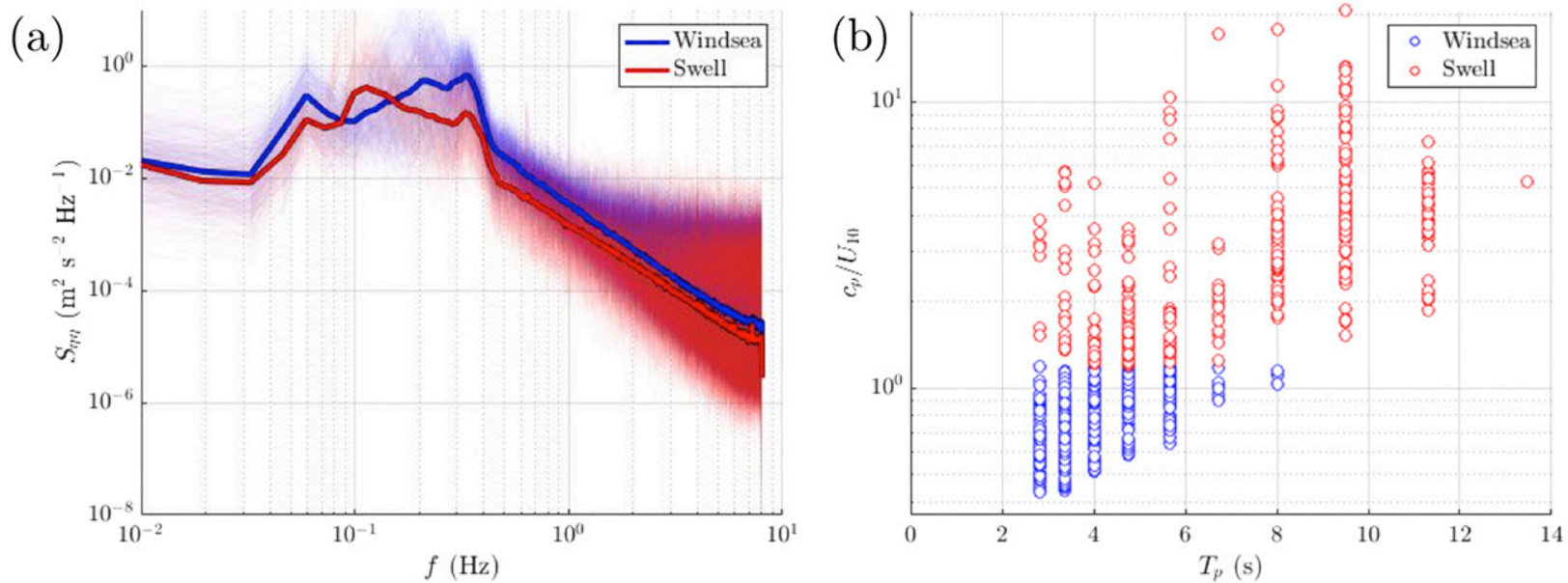

FIG. 4. Sea conditions observed during LASER. (a) Individual (gray lines) and per-frequency-bin median water surface elevation spectra for wind-sea and swell conditions constructed from Senix ultrasonic distance meter measurements. (b) Distributions of observed peak wave periods relative to the Pierson and Moskowitz (1964) wave age $\beta=c_{p} U_{10 \mathrm{~N}}^{-1}$.

wavenumber, frequency, and period. The phase speed of the dominant wave is

$$
c_{p}=g T_{p} / 2 \pi
$$

and finally, the wave age is

$$
\beta=c_{p} / U_{10 \mathrm{~N}}
$$

Figure 4a shows the median water surface elevation spectra $S_{\eta \eta}$ for wind-sea and swell conditions, while Fig. $4 \mathrm{~b}$ presents a summary of the sea conditions observed during LASER according to their wave age and peak period.

(a)

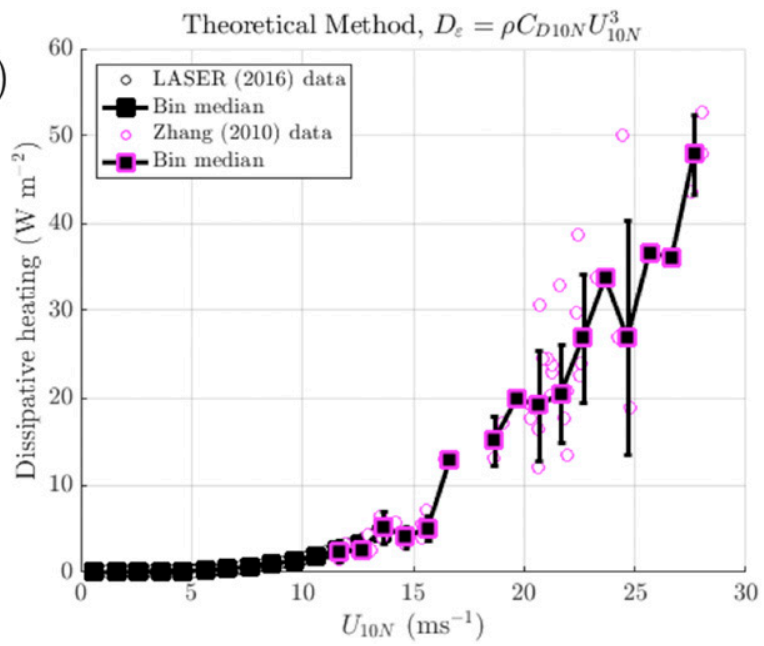

\section{Results}

\section{a. Dissipative heating measurements and a dissipation-drag relationship}

We first present the fundamental and motivating result of dissipative heating measurements, which we have calculated using the original equation of BE98's (4b), as well as the dissipation-rate-dependent equation put forth in ZH10's (9). The dissipative heating values obtained with these equations are shown as a function of 10-m neutral wind speed in Figs. 5a and 5b, respectively. The 10-m wind speeds observed including our data with that of ZH10 span a range from $0.25-28.5 \mathrm{~m} \mathrm{~s}^{-1}$, from calm to full-gale Beaufort-scale conditions.

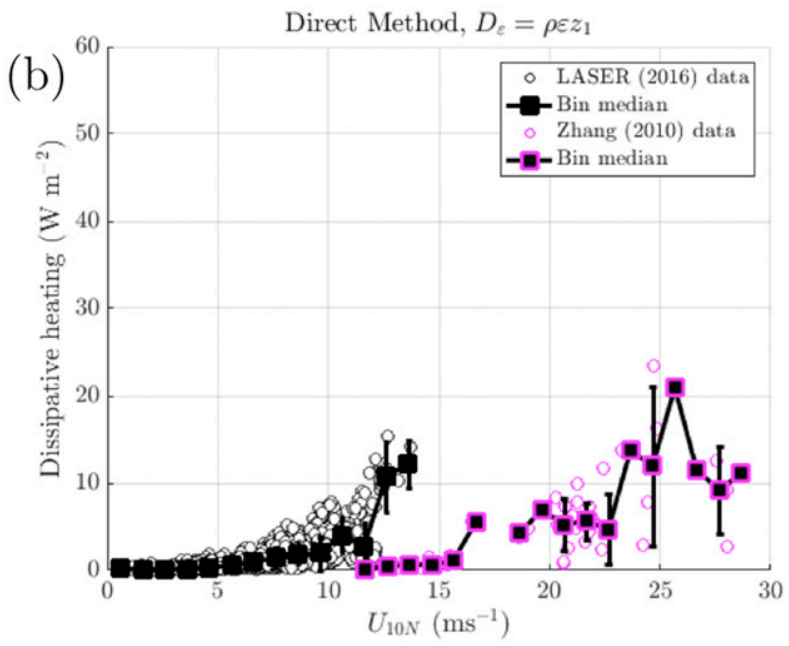

FIG. 5. Dissipative heating $\left(\mathrm{W} \mathrm{m}^{-2}\right)$ measurements as a function of $U_{10 \mathrm{~N}}$ using the equation from (a) BE98 and (b) ZH10. In both panels, the black and magenta points indicate data from the current study and from ZH10, respectively. Black lines with squares indicate the median of binned data, with error bars denoting plus and minus one standard deviation. 
Dissipative heating generally increases with wind speed regardless of the computational method used; however, the behavior as a function of wind speed is noticeably different. In Fig. 5a, dissipative heating magnitude increases from 0 to $52.5 \mathrm{~W} \mathrm{~m}^{-2}$ and the curve follows a continuous power-law shape with an overall positive trend. Responding to the explicit dependence on wind speed of the BE98 dissipative heating equation, the dissipative heating magnitude increases very rapidly and is substantially larger in the hurricane environment of ZH10 than in the nonhurricane environment of LASER (2016), which we investigated. Conversely, in Fig. 5b, both sets of data measured dissipative heating values between 0 and $12.5 \mathrm{~W} \mathrm{~m}^{-2}$. Furthermore, the dissipative heating does not increase continuously throughout the range of observed wind speeds in the manner of Fig. 5a, but rather the dissipative heating reaches a peak of 10.25 and $20.5 \mathrm{~W} \mathrm{~m}^{-2}$ in the two datasets, respectively, and then decreases thereafter. We use the same moist air density $\rho$ to calculate dissipative heating in the two equations, and the prescribed surfacelayer height is a scalar that would only alter the magnitudes via linear multiples. Hence, the dissipation rate of TKE over the atmospheric surface layer is governing the dissipative heating magnitude in a nonlinear way and the amount of heating appears physically constrained. Although the comparison in Fig. 5 is limited by the maximum 10-m wind speeds observed during LASER (2016) and by ZH10, which do not reach the highest wind speeds seen in a TC (i.e., $96.11 \mathrm{~m} \mathrm{~s}^{-1}$ by Hurricane Patricia in 2015), it is next important to explain why the use of ZH10's direct computation is more appropriate for determining the dissipative heating.

To do so, we return to the reevaluation of dissipative heating in TCs put forth by Kieu (2015), in which the author presents energy budgets for two atmospheric volumes that compare the effect of inclusion of the ASL. The first volume extends from the top of the ASL, denoted $z=h$ to the height of the top bound surface $z=H_{t}$, and this domain was used in E86; the second volume includes the ASL and extends from the surface $z=0$ to $z=H_{t}$ [see Kieu (2015)'s Fig. 2]. The energy budget into the atmospheric volume extending over the PBL for $z \in\left[h, H_{t}\right]$ is simply $\left|Q_{\text {in }}\right|-\left|Q_{\text {out }}\right|=\left|F_{\text {diss }}\right|$, where $\left|Q_{\text {in }}\right|=2 \pi C_{k} \int_{0}^{R_{0}} \rho\left(k_{s}^{*}-k_{h}\right)\left|V_{s}\right| r d r$ is the enthalpy input at the lower surface of the domain, $\left|Q_{\text {out }}\right|=$ $(1-\eta)\left|Q_{\text {in }}\right|$ is the outflow of energy at the top surface of the domain, and $F_{\text {diss }}$ is the frictional dissipation within the volume due to internal eddy viscosity defined in (7a). Here $\eta=\left(T_{s}-T_{o}\right) / T_{s}$ is the Carnot efficiency. Therefore, in the well-mixed PBL domain the dissipative heating effects are entirely contained in the frictional dissipation within the atmospheric volume without providing an extra heat source. When the domain is extended by BE98 to include the ASL however, assumptions are made that while valid in an assumed wellmixed PBL are not valid for the ASL.

BE98 used a gradient wind balance, radial momentum, and thermodynamic equation in their derivation of dissipative heating. Explicitly, the behavior of the angular momentum $R^{2}$ and equivalent potential temperature $\theta_{e}$ are valid for $z \in[h, H]$ - that is, over the well-mixed PBL; however, these are extended to include the domain $z \in[0, h]$, where a constant wind speed with height contradicts the shear included in the BE98 formulation of dissipative heating [see e.g., BE98's (4)]. The second assumption that the wind speed is exactly zero at the air-sea interface $(z=0)$ implies that the ocean surface feels no stress from the wind in the ASL, and no work is exerted upon it therefore by the wind and no momentum is being communicated to the ocean or waves. Such a situation is inconsistent with what we observe in reality, and therefore the dissipative heating must be some fraction of the total frictional work imposed on the ASL by the overlying PBL, while the majority of the energy is transferred to surface waves and currents. Alternatively, ZH10 directly uses dissipation rate estimates from turbulent velocity measurements valid in the surface layer $z \in\left[0, z_{1}\right]$ and the degree to which the dissipation rate increases with increasing wind speed is therefore based entirely on the effectiveness of frictionally induced shear and buoyant processes, without the prescribed linear vertical wind shear or zero surface velocity assumptions in BE98. To determine whether the dissipative heating is physically constrained, we examined how the dissipation rate is related to the aerodynamic drag coefficient $C_{D 10 \mathrm{~N}}$.

The drag coefficient-dissipation rate relationship is examined in Fig. 6, using measurements (Fig. 6a) exclusively from LASER, in both wind-sea and swell conditions, and (Figs. 6b,c) combining measurements from LASER and ZH10 to extend the range of drag coefficient and TKE dissipation rate values. The drag coefficient is a ratio of density-normalized frictional stress and mean-flow kinetic energy that describes the efficiency of momentum transfer across the air-sea interface; it is physically constrained by the structure of the sea surface subject to wind stress. Physical saturation of the air-sea interface occurs when additional stress can no longer increase the effectiveness of energy transfer, likely due to the collapse of the sea surface structure in high-wind conditions (Donelan et al. 2004). The dissipation rate of TKE is found to positively respond to increasing sea surface roughness associated with a larger drag coefficient; however, our observations do limit the robustness of the response for $C_{D 10 \mathrm{~N}}>1.35 \times 10^{-3}$. By 

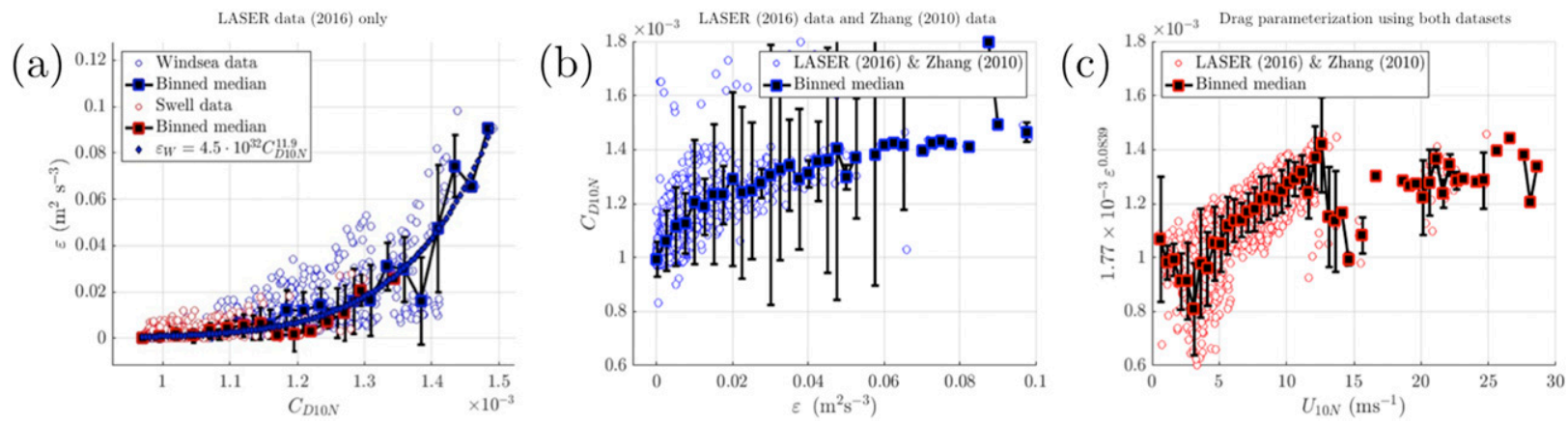

FIG. 6. (a) TKE dissipation rate $\varepsilon$ as a function of 10-m neutral drag coefficient $C_{D 10 \mathrm{~N}}$ for wind sea (blue) and swell (red) from LASER (2016). The blue diamond line is the power-law best-fit line. Black lines with colored squares indicate median of binned data, with error bars denoting plus and minus one standard deviation. (b) Drag coefficient $C_{D 10 \mathrm{~N}}$ relationship with $\varepsilon$ based on combined LASER and ZH10 data. (c) Drag coefficient $C_{D 10 \mathrm{~N}}$ parameterized using $\varepsilon$ vs combined $U_{10 \mathrm{~N}}$ measurements from LASER and ZH10.

curve fitting the median of the binned dissipation rates in (6b), we find that the TKE dissipation rate fits a power-law relationship with the drag coefficient for wind sea,

$$
\varepsilon_{W} \approx 4.5 \times 10^{32} C_{D 10 \mathrm{~N}}^{11.9}
$$

with $R^{2}=0.93$ and $\mathrm{RMSE}=7.1 \times 10^{-3}$. As drag coefficient measurements for swell were confined to a much smaller range of values, we omit fitting these data to a curve that would be constructed with sparse data at larger drag values. Independent of sea state, by combining the LASER and ZH10 dissipation rate, drag coefficient, and wind speed measurements, we are able to parameterize the drag coefficient using the dissipation rate; thereafter, the parameterized 10-m drag exhibits the smooth to rough transition and the approach toward saturation seen in conventional measurements of the drag coefficient (Fig. 6c). Additionally, by determining an empirical parameterization for the dissipation rate in terms of a common and simple metric like the 10-m drag coefficient, a relationship between the structure of the air-sea interface itself and in part the behavior of the TKE budget, also suitable for use in numerical models, is now readily accessible.

\section{b. Stability dependence of dissipation rates}

While the efficiency of momentum transfer increases for local wind sea in roughening conditions, the atmospheric stability can suppress TKE production and its rate of dissipation. The Monin-Obukhov stability parameter $\zeta$ is defined as the ratio of shear and buoyancy production; however, shear always contributes to TKE gain so the eddy virtual potential temperature flux dictates whether stable or unstable conditions are present. Figure 7 presents a time series of the entire LASER experimental record (19 January-13 February) of the ratio of dissipative heating to sensible and latent heat flux (Fig. 7a), the atmospheric stability (Fig. 7b), and 10-m neutral wind speed (Fig. 7c). Although sensible and latent heat fluxes are usually much larger in magnitude than the dissipative heating term, we can see under certain circumstances that the dissipative heating and therefore the ratio in Fig. 7a may be large. Yellowhighlighted regions in the time series show areas where the ratio of dissipative heating and heat flux is greater than $0.10(10 \%)$. Within these yellow regions, we can see that large ratio values $(0.2-0.8+)$, or where dissipationdriven heating is large, the atmospheric surface layer is either neutrally stable or weakly stable. Furthermore, the dissipative heating to heat flux ratio does not appear to respond to changes in the $10-\mathrm{m}$ neutral wind speed. We also remind the reader that dissipative heating is an internal consistency and ought to be parameterized at the top of the atmospheric surface layer as part of the overall enthalpy flux, not as an external heat source to the atmosphere but a consequence within the boundary layer of the loss of kinetic energy.

We can place the stability dependence of the dissipation rate in the context of the overall TKE budget by examining similarity relationships. Normalized structure functions, dependent on Monin-Obukhov stability parameter alone, describe the behavior of each term in stable, neutral, and unstable atmospheric surface layers (e.g., Wyngaard and Coté 1971), and are derived by taking the product of all right-hand side terms from (8) with $\kappa z / u_{*}^{3}$. Dimensionless structure functions mathematically consistent with the stable and unstable $\zeta$ regimes in Edson and Fairall (1998) are plotted in Fig. 8.

Partitioning the data into wind sea and swell, it is immediately clear that swell does not have any dependence on the Monin-Obukhov stability; however, interestingly observations in the stable regime were almost entirely comprised of swell conditions. In stable 
(a)

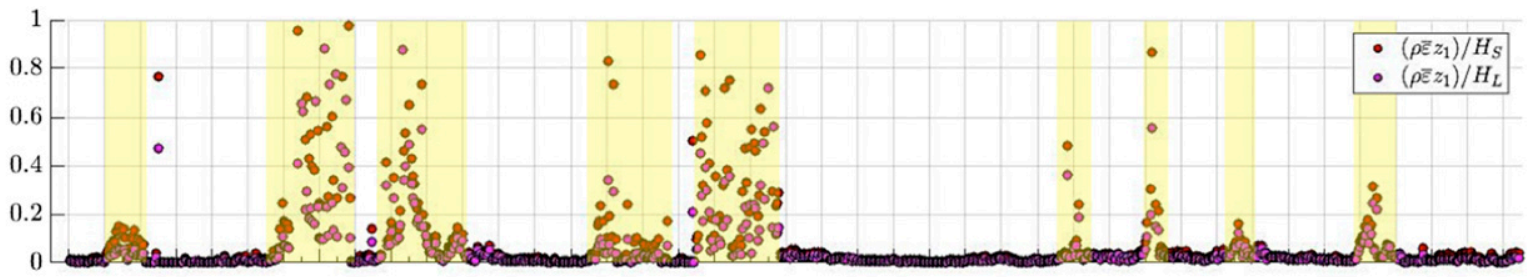

(b)

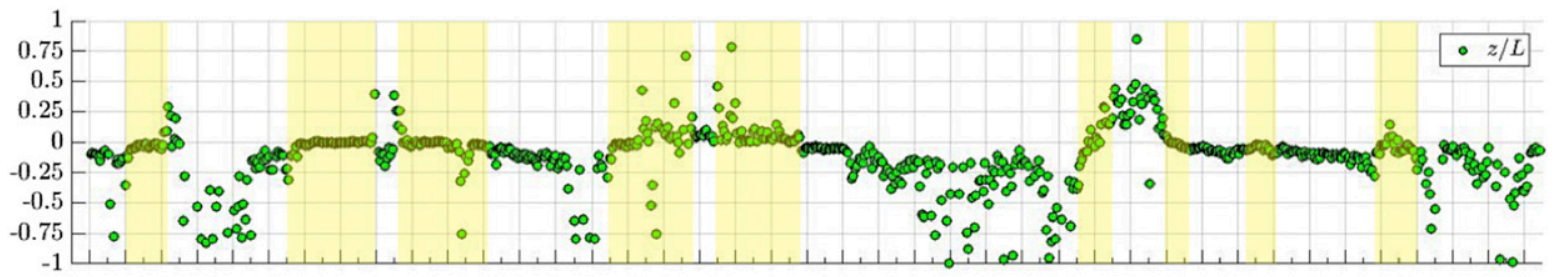

(c)

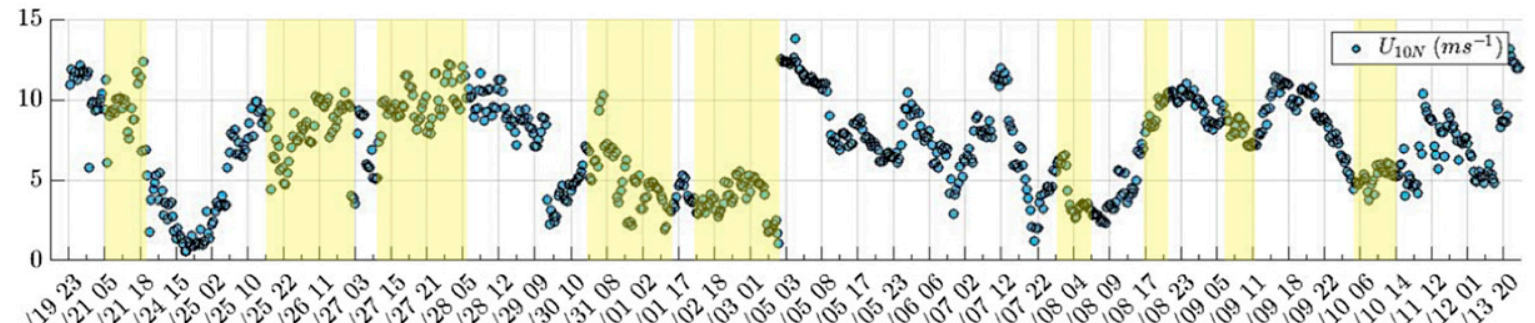

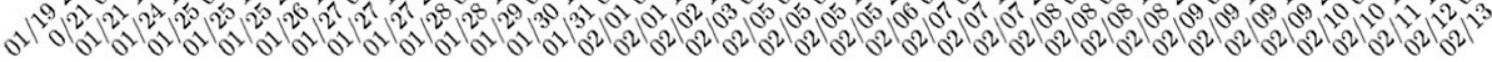

Date/Time (YY/MM HH UTC)

FIG. 7. (a) Ratio of dissipative heating and sensible (red) and latent (magenta) heat flux, (b) Monin-Obukhov stability, and (c) 10-m neutral wind speed $U_{10 \mathrm{~N}}$ time series during LASER from 19 January to 13 February 2016. Yellow-highlighted regions denote areas where the ratio of dissipative heating to heat fluxes is larger than $10 \%$.

conditions, TKE production by shear balances dissipation, while the negative buoyancy suppresses TKE; hence, a net negative TKE production is observed without substantial upward transport. With vertical velocities small and near zero in near-neutral stability, we surmise that the consequential reduction in enthalpy fluxes explains the large dissipative heating to enthalpy flux ratios observed in such stability conditions. By contrast, during LASER we observed predominantly neutral or unstable conditions. Buoyancy production of TKE by convection-induced mixing of the surface layer is the strongest contributor to the energy budget in these conditions, although shear production does still occur. Turbulent transport and pressure work are nonnegligible as instability increases; the turbulent transport of TKE upward results in a local loss of TKE while the local loss of mass induces negative pressure perturbations and a positive pressure work term overall. Hence, the TKE dissipation rate is largest, that is, contributes most negatively to the amount of local TKE, in nonneutral stability away from zero. Additionally, we surmise that the large values of the ratio of dissipative heating to heat fluxes in Fig. 7a reflect small values of the eddy heat flux in near-neutral stability conditions. In such circumstances, the vertical velocities would be small or near zero.

\section{c. Sea-state influence on dissipation rates}

Efficient momentum transfer and nonneutral atmospheric stability have been shown to improve or encourage the rate of TKE dissipation over the open ocean during LASER (Figs. 6 and 7). However, delving further into the relationship between dissipation and drag, we specifically investigated the role of steepness and wave age on the TKE dissipation rate. In Fig. 9, we compare the wave steepness (Figs. 9a,b) and wave age (Figs. 9c,d) of observed wind-sea and swell conditions during LASER. Wave steepness $\delta=H_{\text {sig }} 2 \pi / g T_{p}^{2}$ is the ratio of wave height to wavelength. Increasing steepness of wind-sea waves increases the dissipation rate for $\delta=$ 0.05-0.132 approaching a maximum dissipation rate of $\varepsilon \approx 2.6 \times 10^{-2}$; this positive trend is shown by a linear fit of $\varepsilon \approx 9.80 \times 10^{-2} \delta+0.0043$ with $95 \%$ confidence intervals. Steeper nonbreaking wind sea may enhance the shear production of TKE by mechanically lifting turbulent air parcels, particularly in positively buoyant 


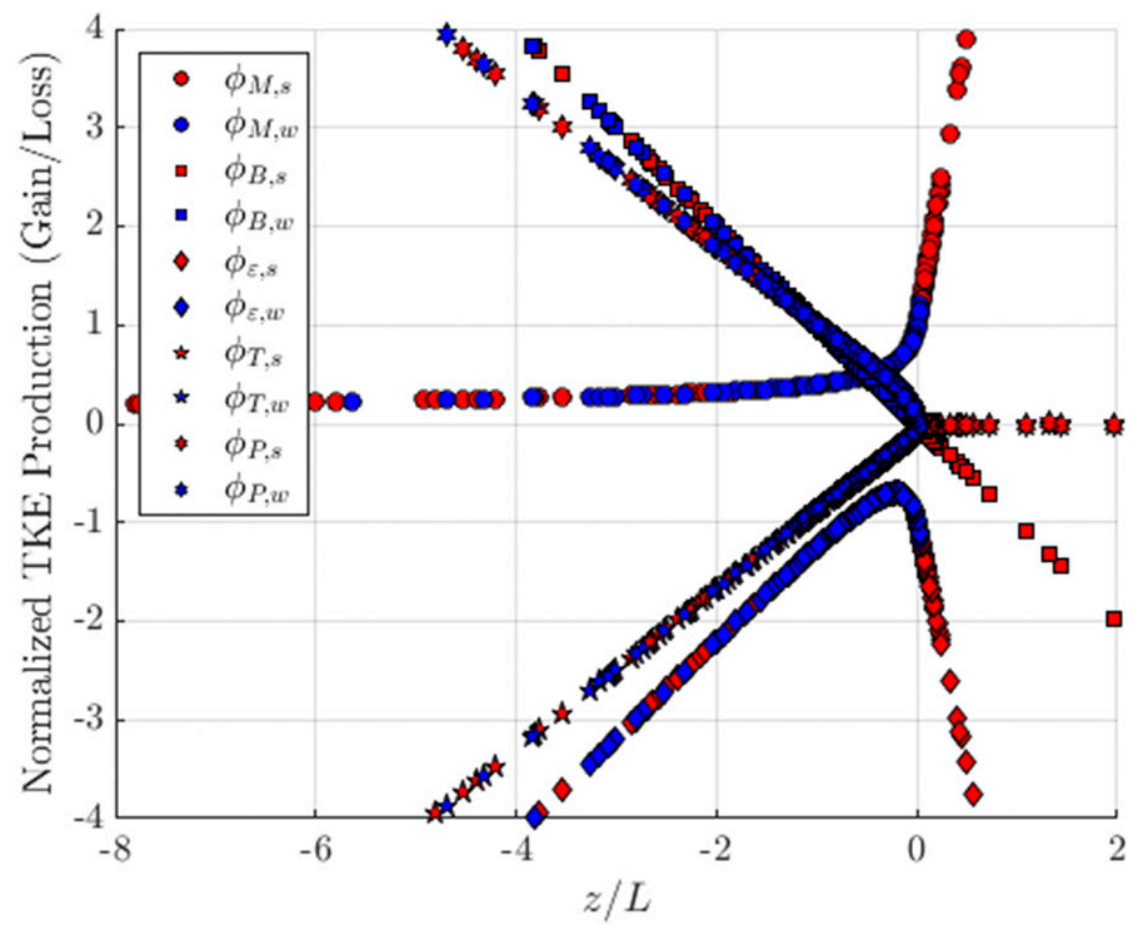

FIG. 8. Normalized TKE budget structure functions as a function of Monin-Obukhov stability $\zeta$ for production, transport, and dissipation terms in (8), following Edson and Fairall (1998). Blue markers and $w$ subscripts denote wind sea, while red markers and $s$ denote swell.

atmosphere (i.e., a form drag effect), while being a steeper wave is also more energetic and the surface and crest of waves are typically rougher (i.e., a skin drag effect). Swell waves, although independent of the local wind could also have structure and shape that may influence dissipation. However, for swell the rate of dissipation increases only for steepness $\delta \approx 0.01-0.05$ with a maximum of $\varepsilon=3.1 \times 10^{-3}$, after which the rate levels off and then, decreases. Furthermore, the slope of the linear fit for these waves is only $\partial \varepsilon / \partial \delta \approx 1.19 \times 10^{-3}$. We speculate that in other regions of the Gulf of Mexico, the likely generation site for these swells, the sea surface was subjected to limited wind stress from low-wind conditions and consequentially the swell we observed possessed long wavelengths and small wave heights; therefore, more vigorous swells could be expected to be more influential on the dissipation rate (e.g., by buoyant production of TKE). Overall, steeper but nonbreaking waves likely produce larger dissipation rates by mechanically enhancing turbulent vertical velocities through form-drag and skin-drag-like effects.

Given the proposed mechanism for enhanced TKE dissipation with steepening of waves, and the fact that the Pierson and Moskowitz (1964) criterion for wave age is a simple threshold value, an increase in wave age expectantly decreases TKE dissipation rates for both wind sea and swell. TKE dissipation rates for the wind-sea waves begin large $\left(\varepsilon \approx 1.3-1.4 \times 10^{-2}\right)$, but decrease to $\varepsilon=4.1 \times 10^{-3}$ according to the linear fit $\varepsilon \approx-1.31 \times 10^{-2} \delta+0.000206$. For swell, TKE dissipation rates are largest for wave ages closest to $\beta=1.2$ ), with a maximum bin median of $\varepsilon=3.1 \times 10^{-3}$ at $\beta=1.9$. Based on the sample size per bin of wave age, most of the observed swell had a wave age $\beta<5$. The general trend is a very weak decrease in dissipation rate $\left(\partial \varepsilon / \partial \beta \approx-1.17 \times 10^{-4}\right)$ as wave age increases, reaching the global minimum for swell of $\varepsilon=1.3 \times 10^{-4}$ at $\beta=4.7$. Swell waves with large wave age move fast away from the local TKE production by eddy fluxes acting on the mean vertical structure of the atmosphere, and have smaller dissipation rates as a result. Behavior of the dissipation rate at $\beta>7.5$ is substantially obscured by much fewer observations at these larger wave ages. Steeper and younger wind waves hence appear more effective at dissipating TKE in the low to moderate wind environment observed during LASER, while swell is far less effective according to our observations.

\section{d. Eddy flux behavior with increasing wave age}

Turbulent momentum and heat fluxes act on mean properties of the atmosphere to produce or suppress TKE in the budget, aided or limited by the steepness and 
(a)

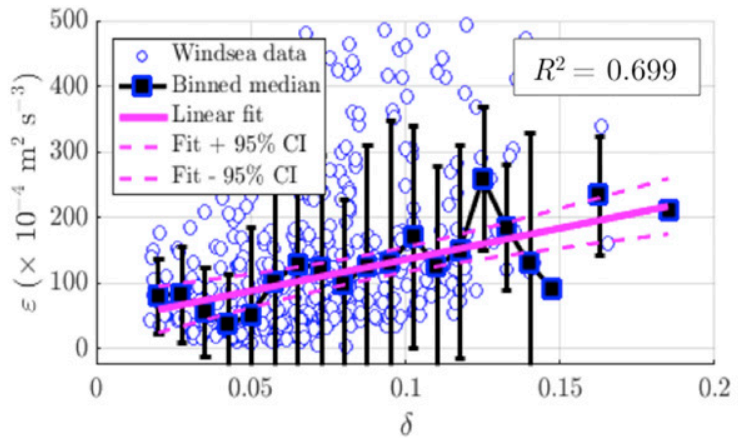

(b)

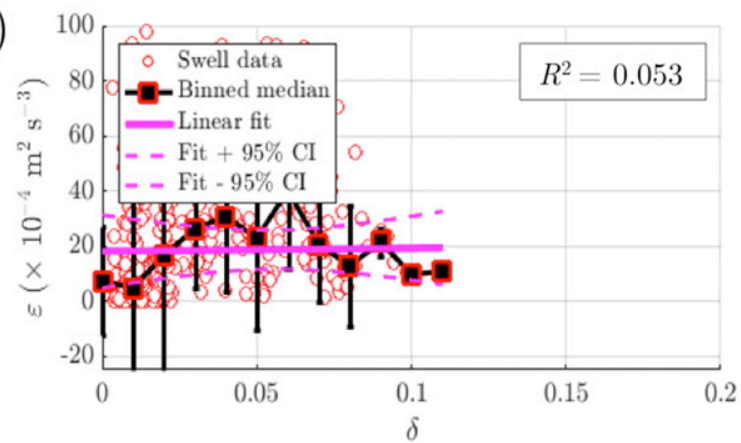

(c)

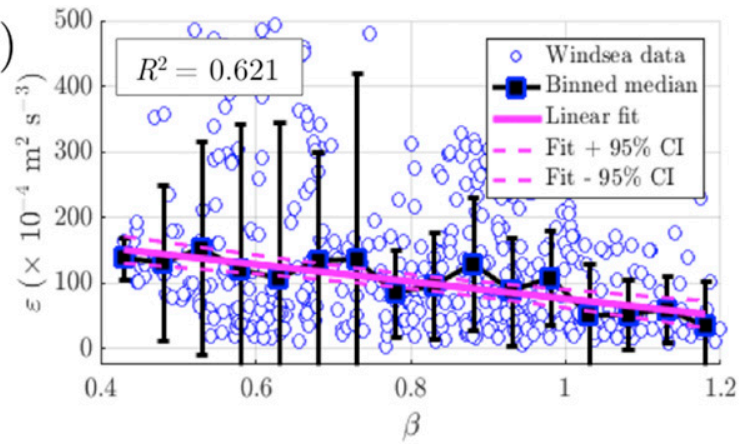

(d)

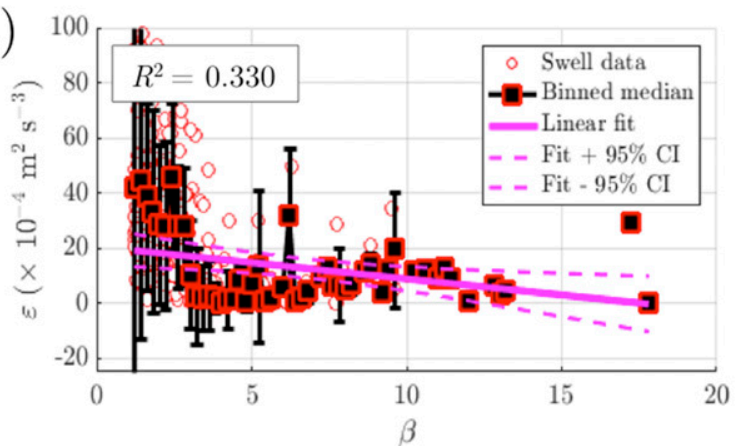

FIG. 9. TKE dissipation rate response to (a),(b) wave steepness and (c),(d) wave age for wind-sea (blue) and swell (red) conditions. Black lines with colored squares indicate median of binned data, with error bars denoting plus and minus one standard deviation. The solid magenta lines are the linear fit, while the $95 \%$ confidence intervals are shown by the dashed magenta lines.

roughness of the sea and its connection to the local wind stress. Following Edson and Fairall (1998), the normalized dissipation was shown to have the largest negative magnitude, that is, highest rate of dissipation by loss of TKE in nonneutral conditions; therefore, nonzero eddy momentum and heat fluxes are important to the air-sea interaction processes contained within the TKE budget. Furthermore, the efficiency of eddy fluxes, specifically momentum flux via the drag coefficient, has been demonstrated to be sensitive to wave age in previous investigations (e.g., Nordeng 1991). Figure 10c shows how $C_{D 10 \mathrm{~N}}$ responds with shifting wave age. Wind sea $(\beta<1.2)$ clearly is more effective at transferring momentum to the surface layer, while the swell is limited to a narrower range of drag coefficient values, with $C_{D 10 \mathrm{~N}} \leq 1.5 \times 10^{-3}$. Swell waves do not appear to have a consistent influence on momentum transfer, with two local minima in $C_{D 10 \mathrm{~N}}$ at $\beta \approx 2$ and $\beta \approx 5.5$. Eddy momentum and heat flux measurements as a function of wave age are presented in Figs. 10a and 10b, respectively. Momentum flux follows the behavior of $C_{D 10 \mathrm{~N}}$, decreasing with wave age consistently for the bulk of the data $(\beta<5)$. The latent heat flux is universally larger than the sensible flux by roughly $20 \mathrm{~W} \mathrm{~m}^{-2}$; however,
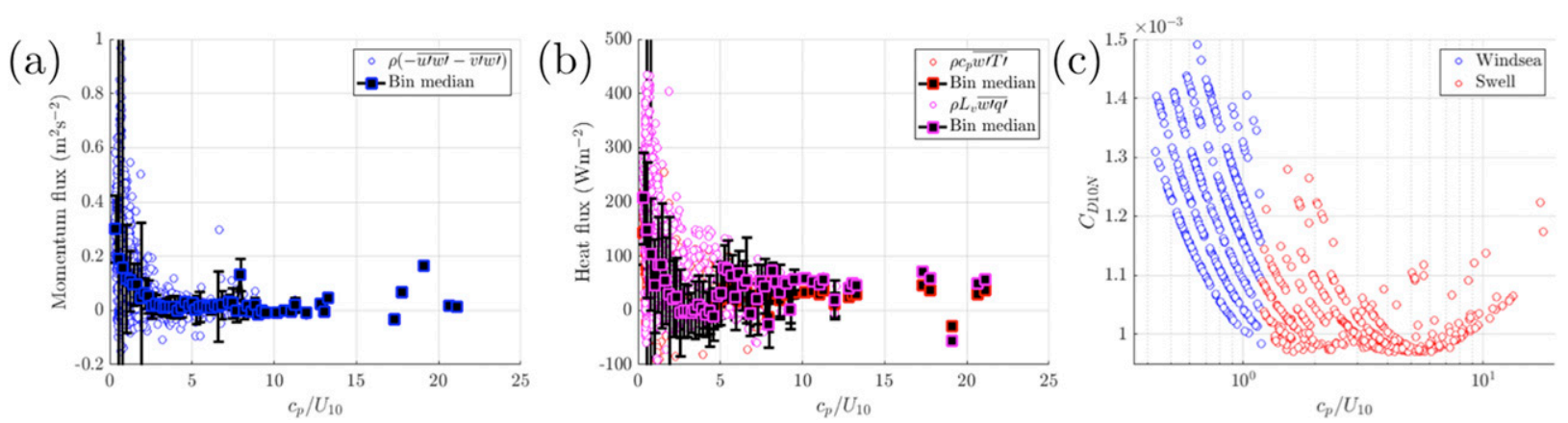

FIG. 10. Wave-age influence on (a) eddy momentum flux, (b) eddy heat fluxes, and (c) 10-m drag coefficient. Black lines with colored squares indicate median of binned data, with error bars denoting plus and minus one standard deviation. The $x$ axis of (c) is plotted in log space for clarity. 
(a)
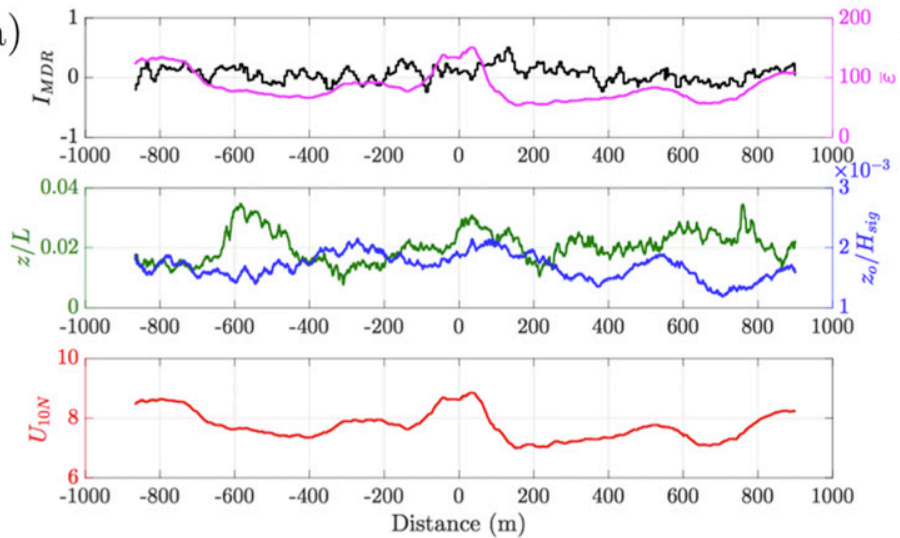

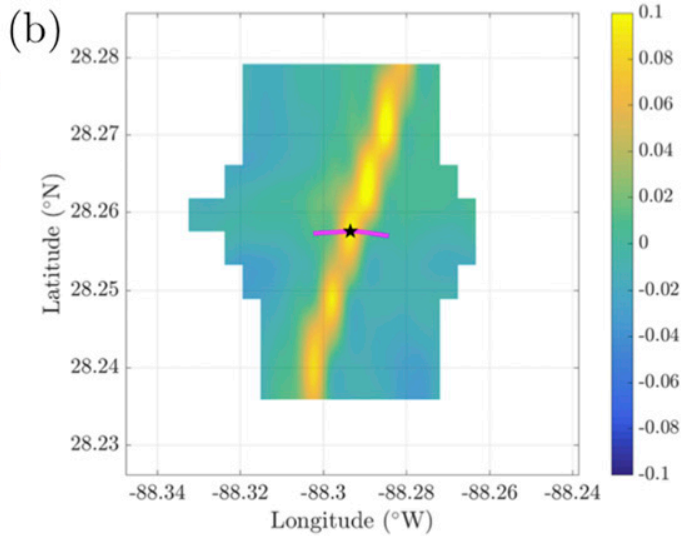

FIG. 11. Cross-frontal structure passing east to west during 0945-1019 UTC 29 Jan. (a) (top) Marine Doppler radar backscatter intensity and dissipation rate, (middle) atmospheric stability and sea surface roughness, and (bottom) 10-m neutral wind speed. (b) A two-dimensional map of the constructed near-surface current shear gradient for the crossing.

both also decrease with wave age for $\beta<5$, from values as much as $435 \mathrm{~W} \mathrm{~m}^{-2}$ to nearly zero. For larger wave ages beyond $\beta=5$, a "detachment" of behavior is noted in the eddy fluxes where neither responds to the wave age in a consistent and predictable manner. Based on the range of wind speeds observed during LASER, the eddy fluxes increase with $U_{10 \mathrm{~N}}$ without saturating; therefore, we believe the wave phase speed $c_{p}$ for wave ages beyond $\beta=5$ produces the variability and inconsistency in the shape of the eddy flux-wave age curves.

\section{e. Dissipation rates near and at surface ocean fronts}

We include a brief mention of intriguing behavior observed during crossings of submesoscale fronts by the R/V F.G. Walton Smith. Near-surface current vectors constructed from marine Doppler radar imagery taken during LASER have shown considerable variability in the amount of shear where surface brightness qualitatively indicates the existence of a surface front. In some cases, strong near-surface current shear was present, and typically associated with a roughness feature slanting as opposed to aligned exactly north-south or west-east. Figure 11 shows such a slanting frontal crossing from 0945 to 1019 UTC 29 January. The cross-front dissipation rate and other properties are given as a function of distance from the front in Fig. 11a, while the nearsurface current shear gradient derived from the current vectors is mapped in Fig. 11b. Strong near-surface current shear at the frontal axis may be associated with more coherent or collocated local maxima in dissipation rate. In some cases, stability and/or sea surface roughness $z_{o} / H_{\text {sig }}$ (cf. Drennan et al. 2005) also had local maxima near or at the front. The level of interaction of the water masses may be indicated by the near-surface current shear, however, it is unclear as of yet how this shear influences either the atmospheric surface layer or the sea surface response, or at what time scale. As suggested in a large-scale investigation of the Kuroshio by D'Asaro et al. (2011), the direction of the mean wind relative to the front's axis may be important in determining near-axis shear but this has not been examined at present. While we have the data necessary to investigate submesoscale frontal crossings during LASER in this context, this is beyond the intended scope of this paper and remains a topic for future study.

\section{Conclusions}

We have used high-frequency ship data from LASER to collect the first reported dissipative heating measurements explicitly in the low-wind, nonhurricane atmospheric boundary layer. The magnitude of the dissipative heating we measured is very similar to aircraft-derived measurements from a high-wind hurricane atmospheric boundary layer observed during the Coupled Boundary Layers Air-Sea Transfer (CBLAST) field experiment, but only when using a dissipative heating formulation directly involving dissipation rate (ZH10) rather than wind speed (BE98). We hence believe the traditional formula in which dissipative heating is proportional to the cube of the wind speed is inappropriate and overestimates the dissipative heating magnitude as suggested by recent studies. A relationship constraining the dissipative heating based on the physical structure of the airsea interface and overlying atmospheric surface layer is much more consistent with our observations, and more readily reconciled with the frictional origin of the phenomenon.

Although roughening of the sea surface is instigated by the wind, by measuring the aerodynamic drag coefficient 
we demonstrate that the dissipation rate follows a power-law relationship with the drag coefficient of $\varepsilon_{W} \approx 4.5 \times 10^{32} C_{D 10 \mathrm{~N}}^{11.9}$ in wind-sea conditions. Dissipation rate, drag coefficient, and wind speed data from LASER and ZH10 were also combined to parameterize $C_{D 10 \mathrm{~N}}$ using $\varepsilon$ for $U_{10 \mathrm{~N}}=0.5-28.5 \mathrm{~m} \mathrm{~s}^{-1}$. The premise of a maximum dissipation rate efficiency for a given drag value presented two dominant processes governing the production of dissipative heating: the atmospheric stability, and the roughness and structure of the sea surface; both determine the efficiency of eddy fluxes of heat and momentum within the atmospheric surface layer. Furthermore, the dissipation process is explicitly involved in the budget of turbulent kinetic energy acting at the interface.

By taking advantage of ship measurements at multiple, fixed vertical levels sampling the boundary layer atmosphere and near-surface ocean, we find dissipative heating can equal $20 \%-80 \%$ of the sensible and latent heat flux in near-neutral or weakly stable stability. Internal TKE loss to dissipative heating (with net-zero energy gain) is therefore competitive with these heat fluxes and not dependent directly upon wind speed. Although a lack of multiple-height pressure measurements only permitted a residual computation of the pressure work, we directly calculated all other TKE budget terms and their normalized structure functions following Edson and Fairall (1998). We determine here that shear (buoyancy) is the dominant mechanism of TKE production, and therefore dissipative heating, in the stable (unstable) atmospheric surface layers sampled during LASER. Nondimensional turbulent transport and pressure work become important as the surface layer becomes more unstable; the turbulent transport of TKE upward results in a local loss of TKE while the local loss of mass induces negative pressure perturbations.

We evaluated the sea state using wave steepness and wave age and found dissipation rates increase with the steepening of wind-sea waves and decrease with wave age overall. Swell waves did not demonstrate strong relationships to the roughness or structure of the sea in the low-wind conditions during LASER. Ultimately, we conclude that dissipative heating will be largest in longfetch environments with nonneutral atmospheric stability characterized by large shear or buoyancy TKE production in stable and unstable conditions, respectively. Steeper wind-sea waves have a larger form drag, resulting in larger eddy momentum and heat fluxes, while slower swell waves have a phase speed closer to frictional stress in the surface layer that may aid their contribution to local shear and buoyancy production, resulting in more dissipative heating. A laboratory experiment to determine the full TKE, heat, and momentum budgets at wind speeds up to $U_{10 \mathrm{~N}}=90 \mathrm{~m} \mathrm{~s}^{-1}$ will be conducted to address the caveats of this study; particularly, we hope to capture the effects of larger swells on buoyancy and pressure terms in the TKE budget, which could be important to the energetics of the hurricane boundary layer.

Acknowledgments. We thank the Consortium for Advanced Research on Hydrocarbon in the Environment (CARTHE) and LASER investigators for collection and management of the raw ship data, Björn Lund for providing marine Doppler radar data, and David G. Ortiz-Suslow for the subsequent data postprocessing. Data are publicly available through the Gulf of Mexico Research Initiative Information and Data Cooperative (GRIIDC) at https://data.gulfresearchinitiative.org (Gulf of Mexico Research Initiative 2018).This work was supported in part by Grant GR009805 from the Gulf of Mexico Research Initiative (GoMRI), as well as by Grant NA14NWS4680028 from the National Oceanic and Atmospheric Administration (NOAA) and Grant AGS1822128 from the National Science Foundation.

\section{REFERENCES}

Anctil, F., M. A. Donelan, W. M. Drennan, and H. C. Graber, 1994: Eddy-correlation measurements of air-sea fluxes from a discus buoy.J. Atmos. Oceanic Technol., 11, 1144-1150, https://doi.org/ 10.1175/1520-0426(1994)011<1144:ECMOAS>2.0.CO;2.

Beron-Vera, F. J., and J. H. LaCasce, 2016: Statistics of simulated and observed pair separations in the Gulf of Mexico. J. Phys. Oceanogr., 46, 2183-2199, https://doi.org/10.1175/JPO-D-15-0127.1.

Bister, M., and K. A. Emanuel, 1998: Dissipative heating and hurricane intensity. Meteor. Atmos. Phys., 65, 233-240, https:// doi.org/10.1007/BF01030791.

Burba, G., and D. Anderson, 2007: Introduction to the Eddy Covariance Method: General Guidelines and Conventional Workflow. LI-COR Biosciences, 141 pp., https://doi.org/10.13140/ RG.2.1.3723.5683.

Businger, S., and J. A. Businger, 2001: Viscous dissipation of turbulence kinetic energy in storms. J. Atmos. Sci., 58, 3793-3796, https://doi.org/10.1175/1520-0469(2001)058<3793: VDOTKE $>2.0 . C O ; 2$.

Curcic, M., 2015: Explicit air-sea momentum exchange in coupled atmosphere-wave-ocean modeling of tropical cyclones. Ph.D. dissertation, University of Miami, https://scholarlyrepository. miami.edu/oa_dissertations/1512.

D'Asaro, E., C. Lee, L. Rainville, R. Harcourt, and L. Thomas, 2011: Enhanced turbulence and energy dissipation at ocean fronts. Science, 332, 318-322, https://doi.org/10.1126/science.1201515.

Donelan, M. A., W. M. Drennan, and A. K. Magnusson, 1996: Nonstationary analysis of the directional properties of propagating waves. J. Phys. Oceanogr., 26, 1901-1914, https://doi.org/ 10.1175/1520-0485(1996)026<1901:NAOTDP > 2.0.CO;2.

, B. K. Haus, N. Reul, W. J. Plant, M. Stiassnie, H. C. Graber, O. B. Brown, and E. S. Saltzman, 2004: On the limiting aerodynamic roughness of the ocean in very strong winds. Geophys. Res. Lett., 31, L18306, https://doi.org/10.1029/2004GL019460. 
Drennan, W. M., P. K. Taylor, and M. J. Yelland, 2005: Parameterizing the sea surface roughness. J. Phys. Oceanogr., 35, 835848, https://doi.org/10.1175/JPO2704.1.

Dugan, J. P., S. L. Panichas, and R. L. Dimarco, 1991: Decontamination of wind measurements from buoys subject to motions in a seaway. J. Atmos. Oceanic Technol., 8, 85-95, https:// doi.org/10.1175/1520-0426(1991)008<0085:DOWMFB >2.0.CO;2.

Edson, J. B., and C. W. Fairall, 1998: Similarity relationships in the marine atmospheric surface layer for terms in the TKE and scalar variance budgets. J. Atmos. Sci., 55, 2311-2328, https://doi.org/ 10.1175/1520-0469(1998)055<2311:SRITMA $>2.0 . C O ; 2$.

Emanuel, K. A., 1986: An air-sea interaction theory for tropical cyclones. Part I: Steady-state maintenance. J. Atmos. Sci., 43, 585-605, https:/doi.org/10.1175/1520-0469(1986)043<0585:AASITF>2.0.CO;2.

Fujitani, T., 1985: Method of turbulent flux measurement on a ship by using a stable platform system. Pap. Meteor. Geophys., 36, 157-170, https://doi.org/10.2467/mripapers.36.157.

Gulf of Mexico Research Initiative 2018: Lagrangian Submesoscale Experiment (LASER) shipboard measurements, northern Gulf of Mexico, January-February 2016.Consortium for Advanced Research on Transport of Hydrocarbon in the Environment II (CARTHE II), accessed 24 January 2017, https://doi.org/10.7266/N7S75DRP.

Högström, U., A. Smedman, E. Sahleé, W. M. Drennan, K. K. Kahma, H. Pettersson, and F. Zhang, 2009: The atmospheric boundary layer during swell: A field study and interpretation of the turbulent kinetic energy budget for high wave ages. J. Atmos. Sci., 66, 2764-2779, https://doi.org/10.1175/2009JAS2973.1.

Holton, J. R., 2004: An Introduction to Dynamic Meteorology. Academic Press, 535 pp.

Jin, Y., W. T. Thompson, S. Wang, and C.-S. Liou, 2007: A numerical study of the effect of dissipative heating on tropical cyclone intensity. Wea. Forecasting, 22, 950-966, https://doi.org/10.1175/WAF1028.1.

Kieu, C., 2015: Revisiting dissipative heating in tropical cyclone maximum potential intensity. Quart. J. Roy. Meteor. Soc., 141, 2497-2504, https://doi.org/10.1002/qj.2534.

Kolmogorov, A. N., 1941: The local structure of turbulence in incompressible viscous fluid for very large Reynolds numbers. Proc. Math. Phys. Sci., 434, 9-13.

Large, W. G., and S. Pond, 1981: Open ocean momentum flux measurements in moderate to strong winds. J. Phys. Oceanogr., 11, 324-336, https://doi.org/10.1175/1520-0485(1981)011<0324: OOMFMI $>2.0 . \mathrm{CO} ; 2$.

Lund, B., H. C. Graber, K. Hessner, and N. J. Williams, 2015: On shipboard marine X-band radar near-surface current "Calibration.” J. Atmos. Oceanic Technol., 32, 1928-1944, https://doi.org/ 10.1175/JTECH-D-14-00175.1.

Miyake, M., M. Donelan, and Y. Mitsuta, 1970: Airborne measurment of turbulent fluxes. J. Geophys. Res., 75, 4506-4518, https://doi.org/10.1029/JC075i024p04506.
Nordeng, T. E., 1991: On the wave age dependent drag coefficient and roughness length at sea. J. Geophys. Res., 96, 7167-7174, https://doi.org/10.1029/90JC02649.

Olascoaga, M. J., and Coauthors, 2013: Drifter motion in the Gulf of Mexico constrained by altimetric Lagrangian coherent structures. Geophys. Res. Lett., 40, 6171-6175, https://doi.org/ 10.1002/2013GL058624.

Pierson, W. J., and L. Moskowitz, 1964: A proposed spectral form for fully developed wind seas based on the similarity theory of S. A. Kitaigorodskii. J. Geophys. Res., 69, 5181-5190, https:// doi.org/10.1029/JZ069i024p05181.

Poje, A. C., and Coauthors, 2014: Submesoscale dispersion in the vicinity of the Deepwater Horizon spill. Proc. Natl. Acad. Sci. USA, 111, 12 693-12 698, https://doi.org/10.1073/pnas.1402452111.

Potter, H., 2015: Swell and the drag coefficient. Ocean Dyn., 65, 375-384, https://doi.org/10.1007/s10236-015-0811-4.

Richman, J., and C. Garrett, 1977: The transfer of energy and momentum by the wind to the surface layer. J. Phys. Oceanogr., 7, 876-881, https://doi.org/10.1175/1520-0485(1977)007<0876: TTOEAM $>2.0 . \mathrm{CO} ; 2$.

Sjöblom, A., and A.-S. Smedman, 2002: The turbulent kinetic energy budget in the marine atmospheric surface layer. J. Geophys. Res., 107, 3142, https://doi.org/10.1029/2001JC001016.

Smith, S. D., 1988: Coefficients for sea surface wind stress, heat flux, and wind profiles as a function of wind speed and temperature. J. Geophys. Res., 93, 15 467-15 472, https://doi.org/10.1029/ JC093iC12p15467.

Sreenivasan, K. R., 1995: On the universality of the Kolmogorov constant. Phys. Fluids, 7, 2778-2784, https://doi.org/10.1063/ 1.868656 .

Von Engeln, A., and J. Teixeira, 2013: A planetary boundary layer height climatology derived from ECMWF reanalysis data. J. Climate, 26, 6575-6590, https://doi.org/10.1175/ JCLI-D-12-00385.1.

Wyngaard, J. C., and O. R. Coté, 1971: The budgets of turbulent kinetic energy and temperature variance in the atmospheric surface layer. J. Atmos. Sci., 28, 190-201, https://doi.org/ 10.1175/1520-0469(1971)028<0190:TBOTKE > 2.0.CO;2.

Zhang, D.-L., and E. Altshuler, 1999: The effects of dissipative heating on hurricane intensity. Mon. Wea. Rev., 127, 3032 3038, https://doi.org/10.1175/1520-0493(1999)127<3032: TEODHO $>2.0 . \mathrm{CO} ; 2$.

Zhang, J. A., 2010: Estimation of dissipative heating using lowlevel in situ aircraft observations in the hurricane boundary layer. J. Atmos. Sci., 67, 1853-1862, https://doi.org/10.1175/ 2010JAS3397.1.

_ _ P. Zhu, F. J. Masters, R. F. Rogers, and F. D. Marks, 2011: On momentum transport and dissipative heating during hurricane landfalls. J. Atmos. Sci., 68, 1397-1404, https://doi.org/10.1175/ JAS-D-10-05018.1. 